This is an author produced version of a paper published in Journal of Economic Dynamics and Control.

This paper has been peer-reviewed but may not include the final publisher proof-corrections or pagination.

Citation for the published paper:

Robert Hart. (2016) Non-renewable resources in the long run. Journal of

Economic Dynamics and Control. Volume: 71, pp 1-20.

http://dx.doi.org/10.1016/j.jedc.2016.07.006.

Access to the published version may require journal subscription.

Published with permission from: Elsevier.

Standard set statement from the publisher:

(c) Elsevier, 2016. This manuscript version is made available under the CC-BY-NC-ND 4.0 license http://creativecommons.org/licenses/by-nc-nd/4.0/

Epsilon Open Archive http://epsilon.slu.se 


\title{
Non-renewable resources in the long run
}

\author{
Rob Hart
}

Department of Economics, Swedish University of Agricultural Sciences, Box 7013, 75007 Uppsala, Sweden.

\begin{abstract}
We model a competitive economy in which production is dependent on labour and a non-renewable resource, the stock of which is inhomogeneous. We solve the model analytically and show how — in infinite time-the economy moves away from an initial balanced growth path (b.g.p.) and towards a mature b.g.p. The characteristics of the initial b.g.p. match historical observations of slowly declining resource price and consumption growth tracking global product. The mature b.g.p. depends on the nature of the stock; the more steeply cross-sectional area declines with depth, the faster the rate of price increase. We show how the theoretical model may be adapted and parameterized to explain and predict the evolution of markets for specific resources, applying the model in two cases, copper and petroleum.
\end{abstract}

Keywords: Non-renewable natural resources, Exhaustible resources, Hotelling rule.

JEL codes: O30; O40; Q31; Q41; Q43.

\section{Introduction}

The optimal exploitation of an exhaustible resource is a classic problem in economics. However, the solution is well-characterized only for a narrow subclass of problems, i.e. those in which the resource stock is homogeneous. In this paper we develop a simple and elegant solution to a much broader class of problems with inhomogeneous resource stocks. In the theoretical model we show how, given the state of the economy at some point in time, we can characterize the paths of extraction, price, and resource rent over infinite time both into the future and the past. We then apply the model to the explanation of historical observations and to the prediction of the future evolution of markets for real resources.

The seminal paper in the resource extraction literature is Hotelling (1931). Hotelling builds a partial equilibrium model focusing on the extraction sector, and shows that - given a finite homogeneous stock of a resource traded on a perfect market - resource price is the sum of unit extraction cost and the scarcity rent, where the latter rises at the discount rate. Furthermore, he demonstrates that the extraction rate is socially optimal as long as markets are competitive.

Dasgupta, Heal, Solow, and Stiglitz — in a series of papers the first of which were published in 1974-apply Hotelling's insight to the question of how an exhaustible resource should be exploited at the general equilibrium level, giving rise to what is now known as the DHSS model. ${ }^{1}$ The model is based on the neoclassical growth model, but with a resource input added to the production function and a Hotelling-type extraction sector. It has spawned a large literature focusing on questions such as the ability of capital to substitute for the exhaustible resource, and the ability of technological progress to compensate for declining resource extraction. ${ }^{2}$

In the baseline version of the DHSS model the production function is Cobb-Douglas, and the resource is extracted from a known non-renewable stock at zero cost. An immediate consequence of the Cobb-Douglas is that the resource factor share is constant, which fits with very long-run observations for aggregate resources such as 'metals' and

\footnotetext{
Email address: rob.hart@slu.se (Rob Hart)

${ }^{1}$ The original papers are Dasgupta and Heal (1974), Solow (1974), Stiglitz (1974).

${ }^{2} \mathrm{~A}$ classic paper on the former question is Hartwick (1977); for a useful survey of the latter see Groth (2007).
} 
(a) Global Metals

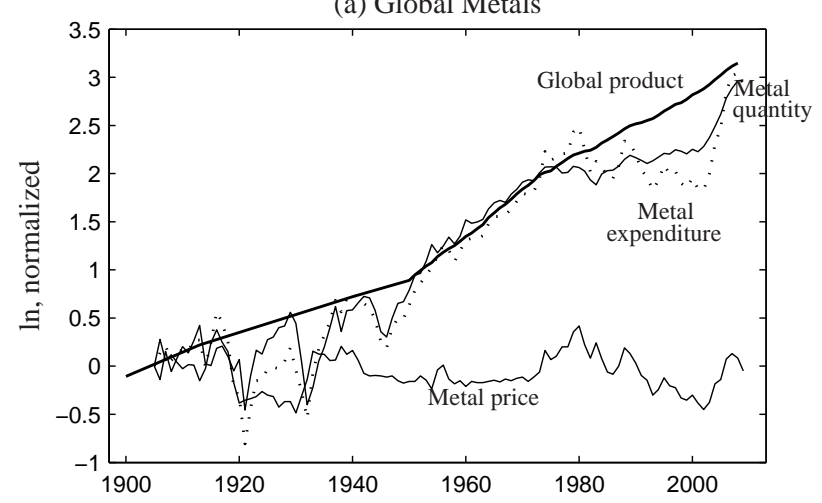

(b) Global Energy

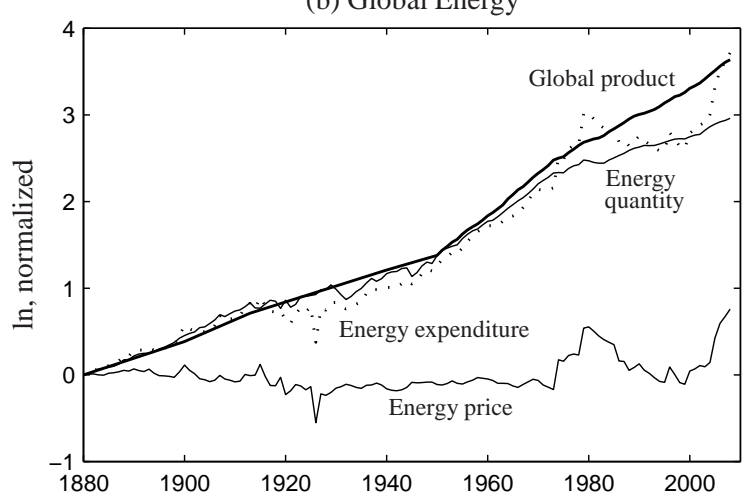

Figure 1: Long-run growth in consumption and prices, compared to growth in global product (i.e. world GDP), for (a) Metals, and (b) Primary energy from combustion.

Note: Global product data from Maddison (2010). Metals: Al, Cr, Cu, Au, Fe, Pb, Mg, Mn, Hg, Ni, Pt, Rare earths, Ag, Sn, W, Zn. All metals data from Kelly and Matos (2012). Energy: Coal, oil, natural gas, and biofuel. Fossil quantity data from Boden et al. (2012). Oil price data from BP (2012). Coal and gas price data from Fouquet (2011); note that these data are only for average prices in England; we make the (heroic) assumption that weighted average global prices are similar. Biofuel quantity data from Maddison (2003). Biofuel price data from Fouquet (2011); again, we assume that the data are representative for global prices, and we extrapolate from the end of Fouquet's series to the present assuming constant prices. Sensitivity analysis shows that the assumptions are not critical in driving the results.

'energy', as shown in Figure 1. On the other hand, the assumption of zero extraction costs leads directly to the prediction that resource price should rise at the discount rate while the consumption rate declines exponentially. This is totally contrary to the evidence, which is that prices show no long-run trend while consumption rates tend to track long-run GDP (see Figure 1 again).

Stocks of exhaustible resources are in reality inhomogeneous and costly to extract, hence an obvious extension to the model is to include these characteristics. However, this area is relatively unexplored, despite the early contributions from Heal (1976) and Solow and Wan (1976). The resource stocks assumed by Heal and Solow and Wan are both special cases: in the former, long-run extraction is from an infinite homogeneous stock, hence the scarcity rent is zero; in the latter it is from a finite homogeneous stock, hence the scarcity rent grows at the discount rate (the simple Hotelling result). Questions remain regarding the transition paths to these long-run states, but also (more generally) the long-run solution to the case in which remaining stocks are always inhomogeneous. Hence there is a need for a more general model of resource stocks to be incorporated into a general equilibrium model of longrun economic development. The need for such a general equilibrium model is indirectly supported by the analysis of Livernois and Martin (2001), who review related work in partial equilibrium frameworks; Livernois and Martin [p.840] state that their findings can easily be reconciled with 'any kind of behaviour for scarcity rent and price over time', simply by introducing exogenous trends or shocks in variables such as extraction productivity (they could also have mentioned resource demand, or the prices of extraction inputs). In general equilibrium, trends in such variables can be endogenized. ${ }^{3}$

We develop a general equilibrium model of the global economy with competitive markets and very simple models of final-good production and biased technological change - models which are broadly in line with historical data and our understanding of the key processes - and focus our attention on extraction. We assume a representative resource owner with extraction rights to a contiguous mass of the resource underground. The resource owner hires labour

\footnotetext{
${ }^{3}$ Examples of partial-equilibrium models can be found in Levhari and Liviatan (1977), Hanson (1980), Slade (1982), Krulce (1993), and Farzin (1992). Levhari and Liviatan (1977) and Hanson (1980) show that the scarcity rent declines over time, Krulce (1993) derives sufficient conditions for increasing rent, and Farzin (1992) argues that rent may also follow a non-monotonic path. Livernois and Martin (2001) show that the results depend crucially on the nature of the instantaneous net benefit function which can be expressed as $\pi\left(x_{t}, n_{t}\right)$ where $x$ is the extraction rate and $n$ is cumulative extraction. For 'clean' results — in which the scarcity rent is always increasing, and approaches the discount rate - they show that the function should be jointly concave in $x$ and $n$. But in practice the shape of the function will depend on the nature of stocks, and there exist highly plausible stock characteristics — such as lower-grade resources being more abundant than higher-grade resources — which imply that the function will not be concave.
} 
inputs in order to extract the resource, and the rate of extraction per unit of labour depends on the productivity of the input, plus the depth of the marginal resource (an increasing function of cumulative extraction). The alternative employment for labour is in final-good production; there is thus an arbitrage condition between the sectors. Labour productivity in the two sectors grows exogenously at rates that may differ. We specify the functional form of the relationship between depth and the cross-sectional area of the resource stock, which allows us to obtain an analytical solution for the dynamic equilibrium path from any given initial state.

Given the state of the economy at any time $t$ we can derive the optimal path of the economy both forwards and backwards in time from $t$. In the baseline model the economy starts close to an initial b.g.p. on which the extraction rate tracks GDP and resource price is constant or slowly declining: technological change drives up the extraction rate and demand for the resource, but has little effect on price as improved productivity is matched by increases in the wage. As the extraction rate increases, resource depth starts to increase significantly, pushing up extraction costs and braking the rise in the extraction rate. Gradually the economy approaches a 'mature' b.g.p. on which depth increases at a constant rate, and this increase pushes up the resource price and slows the growth rate of consumption. Assuming that the total resource stock is finite (implying a restriction on the parameterization of the stock function) then the extraction rate must decline on the mature b.g.p., implying that the price rises faster than the growth rate of GDP. On the mature b.g.p. the scarcity rent is a constant fraction of the price, and this fraction is a decreasing function of the discount rate.

We go on to consider more general cases in which more complex stock characteristics are allowed. The simplest extension is to allow for a maximum depth beyond which no further stocks exist (or are extractable), and there is no substitute for the resource. In such a case the economy will move from the initial b.g.p. towards the mature b.g.p., but instead of approaching this b.g.p. asymptotically it will at some finite time start to move away from it and approach a Hotelling path along which the resource price rises at the discount rate.

Finally, we apply the model to explaining and predicting the evolution of global markets for copper and petroleum: the case of copper illustrates the power of the model, whereas the case of petroleum also highlights some limitations. In the case of copper the cross-sectional area of the representative resource deposit increases with depth initially, and then declines. When extraction is from the upper part of the stock (up to approximately 2050) price declines slowly and the scarcity rent makes up only a very small part of the price, but once extraction moves to the deeper stocks for which cross-sectional area declines with depth, the price starts to rise and the scarcity rent moves towards 40 percent of the price. In the case of petroleum, stocks are funnel-shaped. Again, the scarcity rent is initially low, but once extraction moves onto the 'pipe' part of the funnel (around 2030) the scarcity rent is around 20 percent of the price. Exhaustion occurs around 2125, and the price path in the final decades depends almost entirely on the price of the backstop.

To place our contribution within the literature on non-renewable resource prices and the Hotelling rule-as reviewed by Livernois (2009) — consider the basic Hotelling model with competitive markets, perfect information, a fixed homogeneous stock of the non-renewable resource, constant technology, and partial equilibrium. We stick with competitive markets and perfect information, but allow for inhomogeneity of the stock and technological change, and put the extraction sector into a general equilibrium context. ${ }^{4}$ The paper can thus been seen as building on the tradition where the countervailing effects of resource degradation and technological change are key: see for instance Slade (1982), Farzin (1992), and Lin et al. (2009). In this literature the most common approach is to write down an extraction cost function in which extraction costs are a decreasing function of technology and an increasing function of cumulative extraction. However, in our general equilibrium model we must explicitly account for the inputs used in the extraction sector, and the natural approach is then to posit an extraction function in which the rate of extraction is a function of technology, input use, and the quality of the marginal resource; extraction costs are then derived from the extraction function, given input prices. By focusing on the quality of the resource currently being extracted rather than cumulative extraction or remaining resources - an approach which follows Slade (1982) but differs from much of the subsequent literature - we focus on the factor that directly affects extraction costs rather than proxies for that

\footnotetext{
${ }^{4}$ Clearly resource markets are frequently characterized by both market power and uncertainty about stocks. (Regarding market power, see for instance Ellis and Halvorsen (2002) and Lin (2011).) However, the effects of market power are ambiguous: Stiglitz (1976) shows that in a simple case with constant-elasticity demand there is no difference between the extraction path (and hence also the price path) under monopoly and perfect competition. Furthermore, Arrow and Chang (1982) show that uncertainty about stocks should lead to fluctuation around the price trend rather than changing that trend.
} 
factor. Furthermore, in general equilibrium it becomes clear that technological progress does not automatically reduce extraction costs since overall technological progress raises both the price and the productivity of extraction inputs. ${ }^{5}$ Finally, and crucially, our general equilibrium approach allows us to endogenize the demand function for the resource, which affects the extraction rate and - given our stock function - the rate of change of the quality of the marginal resource, and hence extraction costs.

Two papers which share some of the aims of this paper are Lin and Wagner (2007) (on non-renewable resource extraction in general) and André and Smulders (2014) (on oil extraction). However, our treatment of resource stocks and extraction costs is much more general than in these papers: Lin and Wagner simply assume that extraction costs rise iso-elastically with cumulative extraction from an infinite stock, whereas in André and Smulders oil extraction is costless in the sense that no external inputs are used, but a proportion of the oil stock is used up in the extraction process and hence does not reach the market. For a completely different approach to understanding resource-price dynamics see Spiro (2014).

Other relevant papers include Rogner (1997), Mercure and Salas (2012), and Goeller and Weinberg (1978), which are close to this one in the sense that they assess the nature of global resource stocks, linking these assessments to predictions of future price trends. However, in these papers there is no economic model of demand or scarcity rent, hence no quantitative prediction of price or scarcity rent over time. ${ }^{6}$

Our main focus is on the development of resource prices over time; however, the size of the scarcity rent itself is also important for a number of reasons, of which we highlight two (see Hart and Spiro, 2011, for further discussion). Firstly, any policy measure which reduces the scarcity of a resource will cause a fall in the scarcity rent, reducing the price of the resource and raising the extraction rate. The famous result that a constant ad valorem tax has no effect on the extraction path of Hotelling resource (with zero extraction cost) falls into this category. (For the result see Dasgupta and Heal, 1979; for extensions see Ulph and Ulph (1994) and Sinclair (1994).) Secondly, any policy that leads to expectations of lower future demand — such as the announcement of future taxes - will also lower the scarcity rent and hence boost resource consumption in the short run. This is the 'green paradox' of Sinn (2008); see also for instance Di Maria et al. (2012) and Van der Ploeg and Withagen (2012).

The remainder of the paper is organized as follows. In Section 2 we present the general model without specifying the nature of resource stocks. In Section 3 we introduce the model of resource stocks, and we solve the infinitehorizon problem in which resource depth is unbounded and there is no substitute for the resource. In Section 4 we explore solutions to more general types of problem in which substitutes exist and the nature of resource deposits is more complex than the baseline model allows. In Section 5 we parameterize the model for the cases of copper and petroleum. Section 6 concludes.

\section{The general model}

In this section we set out the general model of resource extraction and use, setting up the Hamiltonian of the representative resource owner and deriving necessary conditions for an optimal solution. Note that since markets are competitive in the model we could also set up the social planner's problem to yield the same conditions.

\footnotetext{
${ }^{5}$ Note that the approaches are mathematically equivalent, i.e. we could reformulate the model starting by writing down an extraction cost function in terms of a technology index and cumulative extraction, and from these equations derive the equations of the model, after making explicit what inputs are used in the extraction process. It would then be clear that the technology index in the extraction cost function is actually the ratio of labour productivity in the extraction sector to labour productivity in the final-good sector, so technological progress drives extraction costs down when it is faster in the extraction sector than in the final-good sector.

${ }^{6}$ Rogner (1997) assess global hydrocarbon resources (cumulative stock) as a function of the predicted extraction cost per barrel of oil equivalent at the time of extraction, and Mercure and Salas (2012) perform a similar operation plotting resources against current extraction cost. Goeller and Weinberg (1978) consider the chemical composition of 'demandite' and 'avalloy', which are weighted averages of non-renewable resources and metal alloys respectively. They find - with the exception of fossil fuels and phosphorus - that the major components of demandite and avalloy are effectively inexhaustible, and that there is a great deal of substitutability between alternative elements in most cases. Nevertheless they predict that 'present patterns of use will persist for the next 30 to 50 years' (implying a change anytime now given the date of publication), followed by a transition phase which might last several hundred years, and finishing in the very long run in the 'Age of Substitutability', a steady state based on abundant materials and recycling. An extended version of our model would allow these conclusions - which are more-or-less pulled out of a hat by Goeller and Weinberg — to be probed more deeply.
} 


\subsection{The basic environment}

Total utility $U$ is a linear function of consumption of the aggregate good $Y$ :

$$
U=\int_{0}^{\infty} e^{-\rho t} Y_{t} \mathrm{~d} t
$$

The discount rate is thus constant and equal to $\rho$.

There is a unit continuum of resource owners, each of whom owns identical stocks of the resource; we can thus consider the representative resource owner and her stock. ${ }^{7}$ We define the extraction rate of the representative owner as $x_{t}$ and cumulative extraction as $n_{t}$. We thus have

$$
\dot{n}_{t}=x_{t}
$$

There is a technology parameter $a_{n}$ associated with each unit of resource, reflecting the physical difficulty of extracting that particular unit. We denote this parameter economic depth. The representative owner hires extraction labour $l_{x}$ as the sole input to extraction. The productivity of extraction labour is $a_{x}$, and the resultant flow of extraction $x$ is given by the following equation:

$$
x_{t}=l_{x t} a_{x t} / a_{n t},
$$

where $a_{n t}$ is the economic depth of the unit of resource extracted at time $t$. Due to discounting, resources are always extracted in order of increasing $a_{n}$, hence we can write

$$
a_{n t}=F\left(n_{t}\right),
$$

where $F$ is an increasing function. The function $F$ depends on the nature of the resource stocks, which we discuss further in Section 3, where we also specify a form for $F$.

Resources are sold to price-taking firms producing the final good, total quantity $Y$. There is a unit continuum of such firms, hence (i) we can consider a representative price-taking firm, and (ii) the flow of resource inputs to the representative production firm is equal to the flow of resource output $x$ from the representative extraction firm. The representative firm's production, denoted $y$, is a Cobb-Douglas function of labour and resource inputs, as follows:

$$
y=\left(a_{y} l_{y}\right)^{1-\alpha} x^{\alpha} .
$$

Here $\alpha$ is a parameter between 0 and $1, a_{y}$ is labour productivity in final-good production, and $l_{y}$ and $x$ are the respective quantities of labour and resources used by the representative firm in production. We thus abstract from capital, which simplifies the model at little cost in terms of explanatory power since there is perfect foresight and the discount rate is constant; if capital were included its long-run growth rate would be equal to the growth rate in $Y$, the capital share would be constant, and the model results would not change in essence.

The productivity indices $a_{y}$ and $a_{x}$ grow exogenously at strictly positive rates, and total labour $L$ is exogenous (although it may vary over time):

$$
\dot{a}_{y} / a_{y}=\theta_{a y} ; \quad \dot{a}_{x} / a_{x}=\theta_{a x} ; \quad \dot{L} / L=\theta_{L} ; \quad L=l_{x}+l_{y} .
$$

We now return to the final-good production function in order to find the market-clearing conditions. Define $p$ as the price of extracted resource, and $w$ as the wage, and normalize the price of the final good to unity. Perfect competition in the final goods sector then implies the following equilibrium conditions (after eliminating $l_{y}$ using $\left.L=l_{x}+l_{y}\right)$ :

and

$$
\begin{aligned}
& w=(1-\alpha) y /\left(L-l_{x}\right) \\
\text { and } & p=\alpha y / x . \\
\text { where } & y=a_{y}^{1-\alpha}\left(L-l_{x}\right)^{1-\alpha} x^{\alpha} .
\end{aligned}
$$

\footnotetext{
${ }^{7}$ We make this assumption for conceptual simplicity. All the results follow if we instead assume the same overall characteristics of the stock, but with heterogeneous ownership, as long as there are many owners at all possible resource depths.
} 


\subsection{Characterization of equilibrium}

We are now in a position to specify the representative resource owner's problem more precisely, and to define competitive equilibrium. ${ }^{8}$ The resource-owner's problem is to choose an extraction path $\left[x_{t}\right]_{t=0}^{\infty}$ in order to maximize the net present value of revenue minus cost,

$$
\int_{0}^{\infty} e^{-\rho t}\left(p x-w l_{x}\right) \mathrm{d} t,
$$

subject to the extraction rate (2), the evolution of the stock (equations 1 and 3), market clearing (equations 4-6), and the constant exogenous growth rates of $a_{y}$ and $a_{x}$.

Definition 1. A competitive equilibrium of the model consists of paths of extraction, wage rates, resource prices, and labour allocation such that factor prices are given by (4) and (5), and the representative resource owner maximizes (7) subject to the extraction rate (2), the evolution of the stock ( 1 and 3 ), and factor prices.

Having defined equilibrium we now set about characterizing it. Recall that in the next section we specify the function $F$ and solve the model; here we set up the current-value Hamiltonian with $n$-cumulative extraction - as the state variable, find the (necessary) first-order conditions in $x$ and $n$, and find the dynamic equations in $x, n$, and $a_{n}$ consistent with these conditions.

To write down the Hamiltonian, start with equation (7) and substitute for $l_{x}$ using equation (2), for $a_{n}$ using equation (3), and for $\dot{n}$ using equation (1). Hence

$$
\mathcal{H}=p x-w x F\left(n_{t}\right) / a_{x}-\lambda x,
$$

where $-\lambda$ is defined as the shadow price of cumulative extraction, and hence $\lambda$ is the current-value scarcity rent.

Take the first-order condition in $x$ and use equation (2) to obtain

$$
\lambda=p-w a_{n} / a_{x},
$$

which simply states that the scarcity rent is equal to the difference between price and unit cost. Now define $\gamma$ as the share of unit extraction costs in the price, implying that $1-\gamma$ is the share of the scarcity rent in the price. Thus $\gamma=w l_{x} /(p x)$ and (from 2 and 9)

$$
\lambda a_{x}=w a_{n}(1-\gamma) / \gamma .
$$

Now use equations (1) and (3) to show that

$$
\dot{a}_{n}=\dot{n} F^{\prime}(n)=x F^{\prime}(n),
$$

return to the Hamiltonian and take the first-order condition in $n$, substitute for $F^{\prime}(n)$ using (11) to yield $\lambda / \lambda=\rho-$ $w \dot{a}_{n} /\left(\lambda a_{x}\right)$, and substitute for $\lambda a_{x}$ using (10) to yield

$$
\frac{\dot{\lambda}}{\lambda}=\rho-\frac{\dot{a}_{n}}{a_{n}} \frac{\gamma}{1-\gamma} .
$$

So the growth rate of the scarcity rent is equal to the discount rate minus the growth rate of economic depth $\times$ the weight of extraction costs in the price relative to the scarcity rent.

To help understand this result, and the debate referred to in the introduction, consider an economy with perfect markets, and a resource stock made up of a series of units $R_{i}$ where $i=1, \ldots, m$. The extraction rights to a given

\footnotetext{
${ }^{8}$ Note that the symmetric equilibrium is optimal. To see this, divide the representative resource owner's resources into two equal parts, and assume that the owner has extracted to a greater depth on one part than on the other. In the presence of discounting this can never be optimal, since costs are borne unnecessarily early along the asymmetric path. Since the resource owners have no market power, what holds for the representative owner must also hold across owners; it can never be optimal for one owner to have extracted to a greater depth than the other owners. That is, they extract symmetrically.
} 
unit $R_{i}$ are an asset, and the value (or shadow price) of this asset, $V_{i}$, should rise at the same rate as the value of other assets in the economy, i.e. at the discount rate $\rho$. Furthermore, given a perfect market the price of extracted resource will be the sum of unit extraction cost and $V_{i}$ at the time of extraction. Now define the scarcity rent $\lambda_{t}$ as the value $V$ associated with the unit of resource being extracted at $t$. If the resource stock is homogeneous then $V_{i}$ will be the same for all $i$, and the scarcity rent $\lambda_{t}$ will rise at the discount rate (Hotelling, 1931). On the other hand, if the resource stock is inhomogeneous then the asset values $V_{i}$ associated with different units of the stock will differ, with lower-quality resources having lower value. Since — given competitive markets and discounting — resources are extracted in order of decreasing quality, the effect of inhomogeneity will in general be to slow the rate of rise of the scarcity rent, and its growth rate must be in the interval $(-\infty, \rho) .^{9}$

Now write (9) as $p=\lambda+w a_{n} / a_{x}$, and note that $\lambda=(1-\gamma) p$ while $w a_{n} / a_{x}=\gamma p$. Differentiate w.r.t. $t$ to yield

$$
\frac{\dot{p}}{p}=\frac{\dot{\lambda}}{\lambda}(1-\gamma)+\left(\frac{\dot{a}_{n}}{a_{n}}+\frac{\dot{w}}{w}-\theta_{a x}\right) \gamma,
$$

and use (12) to eliminate $\dot{\lambda}$ and obtain

$$
\dot{p} / p=\rho(1-\gamma)+\left(\dot{w} / w-\theta_{a x}\right) \gamma
$$

This corresponds to the expression found (for instance) in Krautkraemer (1998) (his equation 8): the growth rate of the resource price equals the discount rate $\times$ the rent share plus the growth rate of productivity-adjusted labour costs $\times$ the extraction-cost share. Thus if the resource price is pure rent it grows at the discount rate, whereas if it is pure extraction cost then it tracks extraction costs. Note that the above equations make clear that increasing productivity of extraction inputs does not necessarily lead to downward pressure on the resource price: if these inputs are in limited supply then there may be a countervailing increase in their price, and the overall effect on the resource price will only be negative if the rate of productivity increase $\left(\theta_{a x}\right)$ outstrips the rate of price increase of the input $(\dot{w} / w)$.

Finally we reexpress the above results in a form that makes clear that we have solved for the necessary conditions on the dynamic evolution of the economy (for the derivation see Appendix A.1):

$$
\begin{aligned}
\frac{\dot{x}}{x} & =\frac{l_{x}}{\alpha L}\left(1+\alpha-\frac{l_{x}}{L}\right)\left(\theta_{a x}+\theta_{L}\right)+\left(1-\frac{l_{x}}{L}\right)\left(\frac{l_{x}}{\alpha L}-1\right)\left(\frac{\rho}{1-\alpha}-\left(\theta_{a y}+\theta_{L}\right)\right)-\frac{l_{x}}{L} \frac{\dot{a}_{n}}{a_{n}}, \\
\frac{\dot{n}}{n} & =\frac{x}{n}, \\
\frac{\dot{a}_{n}}{a_{n}} & =\frac{x F^{\prime}(n)}{a_{n}},
\end{aligned}
$$

where $l_{x}=x a_{n} / a_{x}$ (equation 2). Given these equations - and if the function $F$ is known - then if we know $x, n$, and $a_{n}$ at any time $t$ we can solve for the entire equilibrium path of the economy.

\section{The baseline model with a specified resource stock}

In this section we specify the function $F$, which allows us to solve the model analytically and fully characterize the long-run development of the economy. In 3.1 we explain how resource stocks are modelled in the baseline case, in 3.2-3.4 we concentrate on the mathematical solution of the model, and in 3.5 we discuss the economic intuition.

\footnotetext{
${ }^{9}$ To make the point explicitly, consider two units of stock, $R_{1}$ and $R_{2}$, where $R_{2}$ is lower quality than $R_{1}$. At time $t$, their shadow prices are $V_{1}(t)$ and $V_{2}(t)$ respectively, where $V_{1}(t)>V_{2}(t)$. Both units of the stock are tradable assets, hence both $V_{1}(t)$ and $V_{2}(t)$ grow at the discount rate $\rho$, so $V_{1}(t) / V_{2}(t)$ is constant. Now assume the extraction path is such that $R_{1}$ is extracted at time $s$ and $R_{2}$ at time $s+T$. Then $\lambda_{t}$ is $V_{1}(s)$ at time $s$, and $V_{2}(s+T)=V_{2}(s) \cdot e^{\rho T}$ at time $s+T$. Therefore the average growth rate of the scarcity rent across the period is $\rho-(1 / T) \ln \left(V_{1} / V_{2}\right)$, which must be in the interval $(-\infty, \rho)$.
} 


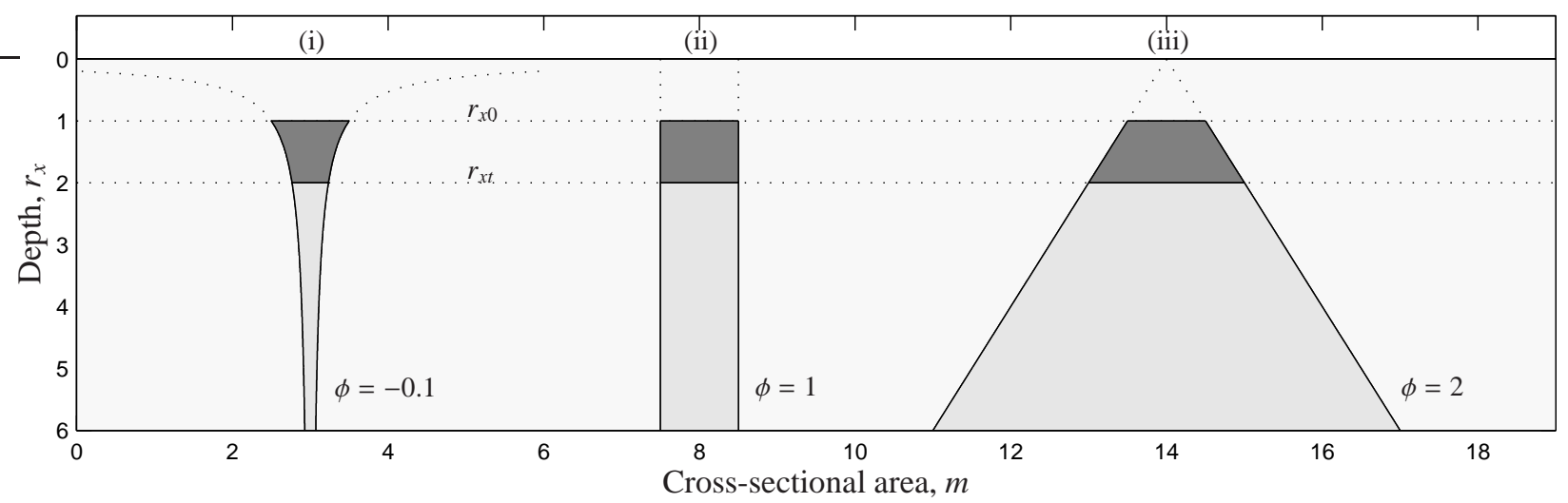

Figure 2: The relationship between depth $r_{x}$ and cross-sectional area $m$ for three alternative resource stocks. In each case $r_{x 0}=1$, $r_{x t}=1$, and $m_{0}=1$. The dark shading represents cumulative extraction at time $t, n_{t}$, and the lighter shading represents remaining resources at time $t$. Resource stocks in the first case are finite, while in cases (ii) and (iii) they are infinite. In Section 4 we analyse more general cases in which stocks are always finite.

\subsection{Resource stocks}

We now assume that the representative owner's stock is a contiguous mass, underground, which extends in space according to the following function:

$$
\frac{m}{m_{0}}=\left(\frac{r_{x}}{r_{x 0}}\right)^{\phi-1} .
$$

Here $r_{x}$ is depth underground ( $r$ for remoteness), $m$ is the cross-sectional area of the resource, $r_{x 0}$ and $m_{0}$ are the depth and cross-sectional area at $t=0$, and $\phi$ is a parameter which may take any (real) value. Although we denote $r_{x}$ as depth, the model can encompass a situation in which the difficulty of extraction is a function of several variables, which may include depth but also grade (i.e. the percentage of the resource by weight in the rock), and the size of the deposit (larger deposits will typically have lower unit extraction costs). In Section 5.3 we give an example of how $r_{x}$ can be related to depth and grade in a specific case, extraction of copper.

The function is flexible, as shown in Figure 2, where three different cases are illustrated. Furthermore, in Sections 4 and 5 we show how a more complex stock may be approximated by building up the total stock piecewise from substocks, and by putting a lower bound on depth. In Section 4 we show how such a case may be solved in theory, and in Section 5 we calibrate the model for copper and petroleum.

Since extraction will always be performed in order of increasing depth, cumulative extraction $n$ is as follows:

$$
n_{t}=\int_{r_{x 0}}^{r_{x t}} m \mathrm{~d} r_{x}
$$

Substitute for $m$ from equation (18) to obtain

$$
n_{t}=\frac{m_{0} r_{x 0}}{\phi}\left[\left(\frac{r_{x t}}{r_{x 0}}\right)^{\phi}-1\right] .
$$

Note that when $\phi \geq 0$ then $n \rightarrow \infty$ when $r_{x} \rightarrow \infty$, so if we want the stock $n$ to be bounded we must impose a limit on $r_{x}$ beyond which there are no further stocks (see Sections 4 and 5); on the other hand, when $\phi<0$ then $r_{x} \rightarrow \infty$ when $n \rightarrow-\left(m_{0} r_{x 0}\right) / \phi$, hence the total available stock at $t=0$ is $-\left(m_{0} r_{x 0}\right) / \phi$. Note also the special case when $\phi=0$, in which case $r_{x} / r_{x 0}=\exp \left(n /\left(m_{0} r_{x 0}\right)\right)$.

Finally, to obtain the function $F$ (recall equation (3), $a_{n t}=F\left(n_{t}\right)$ ) we need to link depth $r_{x}$ to economic depth $a_{n}$. We define the link as follows, also introducing parameters $\psi$ and $F_{0}$ to simplify notation:

$$
a_{n}=r_{x}^{\chi}, \quad \psi=\phi / \chi, \quad F_{0}=m_{0} r_{x 0} / \chi .
$$


Hence (from 20 and 21)

$$
F\left(n_{t}\right)=a_{n t}=a_{n 0}\left(1+\frac{\psi n_{t}}{F_{0}}\right)^{1 / \psi} .
$$

Parameter $\chi$ determines the elasticity of economic depth $a_{n}$ to the physical measure $r_{x}$. For instance, if we interpret $r_{x}$ literally as depth underground, then if depth doubles, unit extraction costs are multiplied by $2^{\chi}$.

\subsection{The steady state}

We now show that, given the above functional form for $F$, there exists a balanced growth path which is consistent with the necessary conditions above $(15,16$, and 17$)$, and that this path may be characterized as a steady state in a new coordinate system. First use (22) to rewrite (17),

$$
\frac{\dot{a}_{n}}{a_{n}}=\frac{x}{F_{0}} \frac{1}{\left(a_{n} / a_{n 0}\right)^{\psi}},
$$

and substitute in to (15) to yield just two dynamic equations. Then choose the following coordinates:

$$
\left(a, l_{x}^{*}\right)=\left(\frac{L}{F_{0}} \frac{a_{x} / a_{n}}{\left(a_{n} / a_{n 0}\right)^{\psi}}, l_{x} / L\right)=\left(\frac{L}{F_{0}} \frac{a_{x} / a_{n}}{\left(a_{n} / a_{n 0}\right)^{\psi}}, \frac{x / L}{a_{x} / a_{n}}\right) .
$$

Note that $a$ is a state variable reflecting the extraction technology in relation to the remaining resource stock (with high $a$ corresponding to easy extraction), whereas $l_{x}^{*}$ (the proportion of labour in extraction) is a control variable. We then have the following proposition.

Proposition 1. The following equations must be satisfied on an equilibrium path:

$$
\begin{aligned}
& \frac{\dot{a}}{a}=\theta_{a x}+\theta_{L}-(1+\psi) a l_{x}^{*}, \\
& \frac{i_{x}^{*}}{l_{x}^{*}}=\left(1-l_{x}^{*}\right)\left[a l_{x}^{*}-\left(1-\frac{l_{x}^{*}}{\alpha}\right)\left(\theta_{a x}-\theta_{a y}+\frac{\rho}{1-\alpha}\right)\right] .
\end{aligned}
$$

Proof. To derive (25) differentiate $a$ w.r.t. time to yield $\dot{a} / a=\theta_{a x}+\theta_{L}-(1+\psi) \dot{a}_{n} / a_{n}$, then substitute for $\dot{a}_{n} / a_{n}$ using (23), and finally use (24). To derive (26) differentiate $l_{x}^{*}$ w.r.t. time to yield $l_{x}^{*} / l_{x}^{*}=\dot{x} / x+\dot{a}_{n} / a_{n}-\theta_{a x}-\theta_{L}$, then substitute for $\dot{x} / x$ using (15) and $\dot{a}_{n} / a_{n}$ using (23), and finally use the definitions of $a$ and $l_{x}^{*}$.

This leads directly to Proposition 2.

Proposition 2. Assume that parameter values are such that

$$
\frac{\rho /(1-\alpha)-\left(\theta_{a y}+\theta_{L}\right)}{\theta_{a x}+\theta_{L}}>\frac{-\psi}{1+\psi} .
$$

Then there is a unique stable steady state to the system described by equations (25) and (26). The steady state is given by the point of intersection of the following curves.

$\dot{a}=0, \quad a=\frac{1}{l_{x}^{*}} \frac{1}{1+\psi}\left(\theta_{a x}+\theta_{L}\right) ;$

$i_{x}^{*}=0, \quad a=\frac{1}{l_{x}^{*}}\left(1-\frac{l_{x}^{*}}{\alpha}\right)\left(\theta_{a x}-\theta_{a y}+\frac{\rho}{1-\alpha}\right)$.

Proof. The proof is straightforward, hence we only sketch it here. To find internal the steady state, set $\dot{a}=0$ and $i_{x}^{*}=0$ in equations (25) and (26), while ruling out corner solutions with either no extraction or no production. To find the restriction on parameter values, first note that when $\psi \leq-1$ the locus of $\dot{a}=0$ is never in the allowed region where both $a$ and $l_{x}$ are positive. Then verify that-for $\psi>-1$ - the locus of $\dot{a}=0$ is always steeper than the locus of $l_{x}^{*}=0$ for given $a$ (and allowed values of $\left(a, l_{x} / L\right)$ ), hence if the lines cross they will do so only once. The condition (which implies that $\psi>-1)$ is necessary and sufficient for the lines to cross. Given that the lines cross it is straightforward to verify that the steady state is stable, for instance with the help of the phase diagram. 

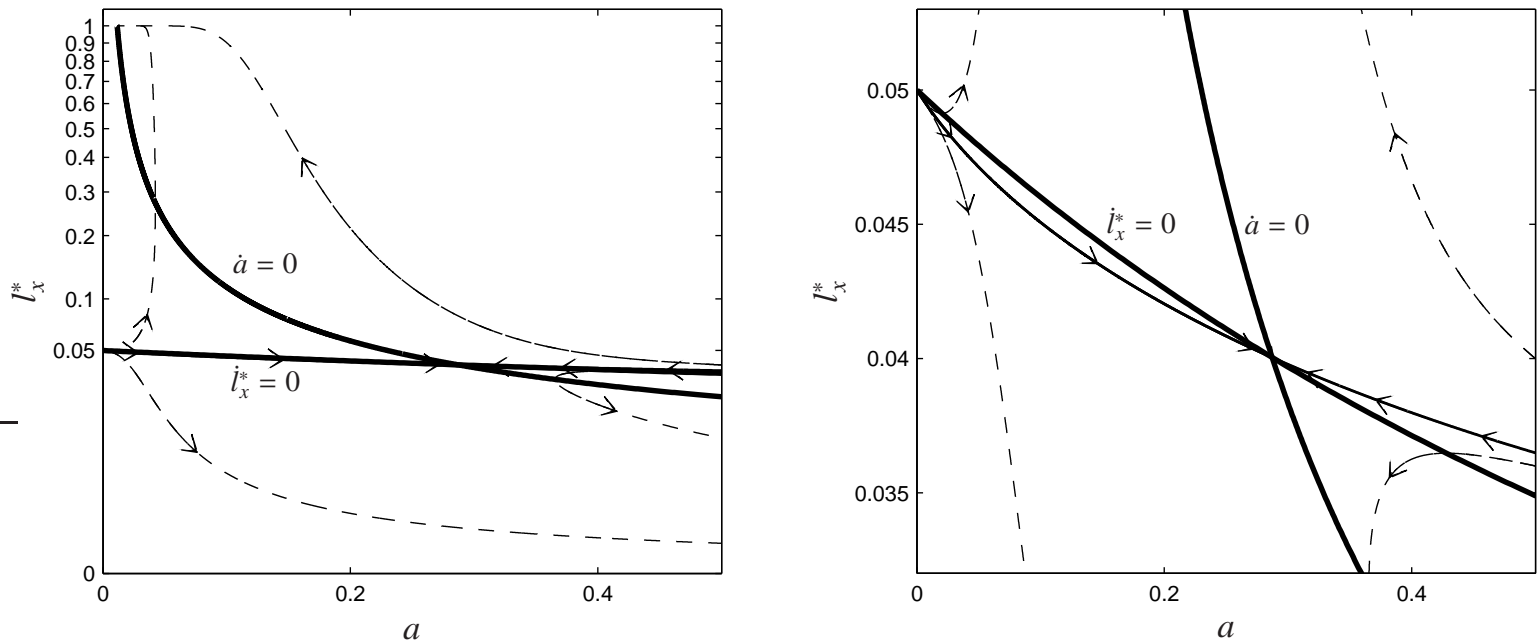

Figure 3: The phase diagram plotted with two different scales on the $l_{x}$-axis: (a) to show the overall characteristics, and (b) to show the stable path.

Given equations (27) and (28) we can construct the phase diagram and characterize the relationship between the initial choice of extraction labour $l_{x}^{*}$ and the subsequent development of the economy: see Figure $3 .^{10}$

\subsection{Transversality}

Having characterized the phase diagram we have characterized sets of paths - from any initial state $a$-all of which are consistent with the necessary conditions (25) and (26). Of these, one set of paths leads to a situation in which all labour is employed in extraction and none in production, and another to a situation in which no labour is employed in extraction. Finally, there is the unique path which leads to the long-run steady state. Before proceeding we should verify that the path leading to the steady state is optimal. We do this using the transversality condition, which is that

$$
\lim _{t \rightarrow \infty} e^{-\rho t} \lambda_{t} n_{t}=0
$$

That is, the present value of resources in the ground (the converse of cumulative extraction) must approach zero looking into the very distant future; if that is not the case it must be possible for the representative resource holder to raise returns by increasing extraction (if the value is positive) or reducing it (if it is negative). For the proof that this transversality condition is a sufficient condition for optimality, and that only the long-run steady state satisfies the transversality condition, see Appendix A.2.

\subsection{The transition path and two b.g.p.s}

Having established that the stable path — as illustrated in Figure 3(b) — is unique and optimal, it is time to characterize it. First note that on any b.g.p. in this economy the following two equations must hold:

$$
\begin{aligned}
& \frac{\dot{y}}{y}=\frac{\dot{w}}{w}+\theta_{L}=(1-\alpha)\left(\theta_{a y}+\theta_{L}\right)+\alpha \frac{\dot{x}}{x} ; \\
& \frac{\dot{p}}{p}=\frac{\dot{w}}{w}+\theta_{L}-\frac{\dot{x}}{x} .
\end{aligned}
$$

These equations follow directly from equations (4)-(6) when we hold labour allocation constant.

Now consider infinite time and recall that $a_{x}$ and $a_{y}$ both grow exogenously at constant rates. So if we move backwards in time then both $a_{x}$ and $a_{y}$ must approach zero. When $a_{x} \rightarrow 0$ then $a \rightarrow 0$ (equation 24), so we approach

${ }^{10}$ Parameter values: $\psi=1, \phi=1, \alpha=0.05, \rho=0.05, \theta_{a x}=0.023, \theta_{a y}=0.0179, L=1$, and $F_{0}=1$. 
(a) $\psi=1$

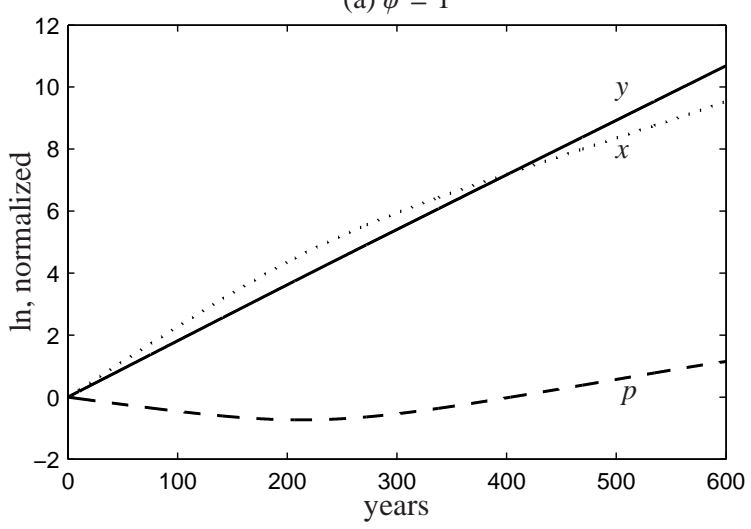

(b) $\psi=-0.4$

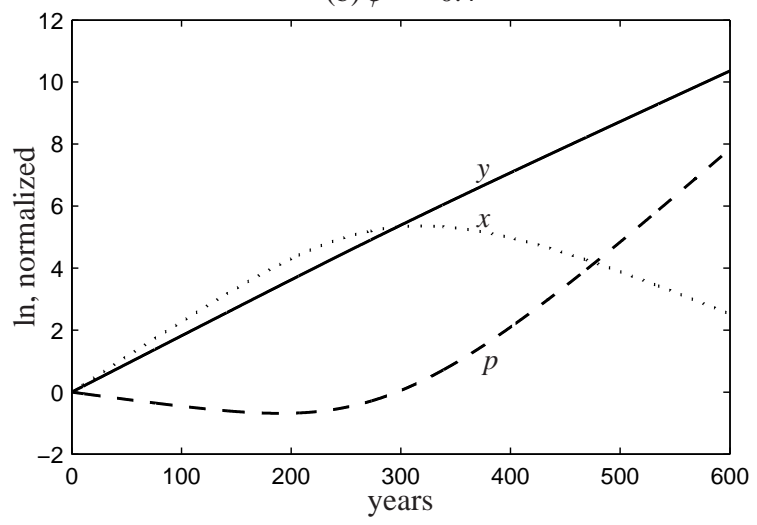

Figure 4: The evolution of $p$ and $x$ compared to $y$ when the initial state $a=10^{-3}$, for alternative values of $\psi$. Other parameters as for Figure 3 . In both cases we see clearly the initial and mature extraction phases, and the transition between them.

the $y$-axis asymptotically moving backwards along the stable path. In accordance with the above reasoning, denote the present time as $t=0$ - with known values of $a$ and $x$ - and assume that the economy at this time is somewhere on the left-hand arm of the stable path illustrated in Figure 3. Now allow $t$ to approach $-\infty$, implying that $a \rightarrow 0$ and $x \rightarrow 0$. It then follows straightforwardly from the representative firm's optimization problem that the economy approaches a b.g.p. going backwards in time. On this initial b.g.p. $\dot{a}_{n} / a_{n}$ and the scarcity rent are both zero, $p$ is simply equal to the extraction cost, and

$$
\dot{x} / x=\theta_{a x}+\theta_{L}:
$$

resource extraction grows at the growth rate of extraction technology plus labour.

Now move forward in time from $t=0$. Then the economy approaches the mature b.g.p. On this b.g.p. we can show (Appendix A.3) that

$$
\text { and } \quad \begin{aligned}
\frac{\dot{x}}{x} & =\frac{\psi}{1+\psi}\left(\theta_{a x}+\theta_{L}\right) \\
\frac{\lambda}{p} & =\frac{\frac{1}{1+\psi}}{\frac{1+(1-\alpha) \psi}{1+\psi}+\frac{\rho-(1-\alpha)\left(\theta_{a y}+\theta_{L}\right)}{\theta_{a x}+\theta_{L}}} .
\end{aligned}
$$

Summing up, in infinite time the economy starts arbitrarily close to the initial b.g.p. on which the scarcity rent is zero and equations (30)-(32) hold. Over time it moves away from this b.g.p. and approaches the mature b.g.p. on which equations (30)-(31) and (33)-(34) hold. The long-run development of the economy - i.e. the transition away from the initial b.g.p. and towards the mature b.g.p. - is illustrated in Figure 4 for two different values of $\psi$, one positive and the other negative.

\subsection{Economic intuition}

Consider a mineral resource such as copper or iron in a very simple closed economy with exogenous technological progress in extraction and final-good production, and exogenous population growth. Assume an initial state with low population and primitive technology, both in extraction and final-good production. The extraction rate is thus very low; the primitive workers are simply scratching the surface of the resources stocks, and depth is almost constant. Moving forwards from this point labour productivity increases, hence the extraction rate increases (equation 32), as does demand for the resource from the final-good sector. The resource price is approximately constant since the productivity increase in final-good production - which drives up the wage - largely cancels out the productivity increase in extraction: from equations (30)-(32) we have $\dot{p} / p=(1-\alpha)\left(\theta_{a y}-\theta_{a x}\right)$. So if labour productivity grows at the same rate in both sectors then the resource price is constant, but if extraction productivity grows faster then the resource price will decline slowly. 
As the extraction rate increases the approximation that $a_{n}$ is constant holds less and less well: depth begins to increase, pushing up the resource price and thus reducing the rate of increase in the quantity demanded in the finalgood sector, and also braking the increase in depth. In the long run the economy approaches the mature b.g.p. on which depth increases at a constant rate. The key equation for understanding the mature b.g.p. is (33), which gives the unique growth rate of extraction that leads to a constant (non-zero) growth rate of depth $a_{n}$. When $\psi>0$, implying an infinite stock, then this growth rate is positive; however, when $\psi<0$ then the stock is finite and the extraction rate declines on the mature b.g.p. In the special case of $\psi=0$ then the extraction rate is constant on the b.g.p. The resource-price trends are opposite to the extraction-rate trends, since the resource share is constant at $\alpha$ : when $\psi=0$ the resource price tracks the overall growth rate (and the wage), when $\psi>0$ price growth is slower than the overall growth rate (Figure 4(a)), and when $\psi<0$ the resource price grows faster than the overall growth rate (Figure 4(b)).

Regarding the scarcity rent, (34) shows that it is a constant fraction of the resource price on the mature b.g.p. Furthermore, the level of the scarcity rent on the b.g.p. (as a fraction of the resource price) is increasing in $\alpha$, and decreasing in $\psi$ and $\rho$. As $\psi \rightarrow \infty$ the scarcity rent on the b.g.p. approaches zero; when $\psi$ is large depth is almost constant and the scarcity rent is very low. On the other hand, in the limit when the inequality in Proposition 2 is satisfied with equality then equation (34) shows that the scarcity rent approaches 100 percent of the price, also implying (equations 12 and 13) that $\dot{\lambda} / \lambda=\dot{p} / p=\rho$; when $\psi$ is negative depth increases rapidly on the b.g.p., implying that current extraction drives up future extraction costs, hence the scarcity rent is high. ${ }^{11}$

The discount rate $\rho$ plays no role in the rate of change of the resource price on the b.g.p. (equations 33-34), by contrast to the classic Hotelling result that $\dot{p} / p=\rho$ for a homogeneous resource with zero extraction cost. However — as shown by equation (34) - the level of the scarcity rent is decreasing in the discount rate. This is because the scarcity rent arises from the fact that today's extraction raises future extraction costs, and the weight of this effect is small when the discount rate is high.

Another way of thinking about the effect of the discount rate is to consider a given state of the system at time $t$ (technology, endowments, resource stock), and assume alternative discount rates. First consider a simple Hotelling model: a resource-dependent economy with a finite stock, zero extraction costs, and a backstop input at a fixed exogenous price $\bar{p}$. Then we know (i) that $p=\lambda$, i.e. the resource price is pure rent, (ii) that $\dot{p} / p=\rho$ (the Hotelling rule), and (iii) that the resource price must be equal to $\bar{p}$ at the point of exhaustion (transversality). A higher discount rate leads to a lower resource price and a higher extraction rate at $t$, and hence a lower growth rate of resource extraction and thus also lower growth in aggregate production. Similar results follow in our model: for a given state of the system at $t$, a higher discount rate implies that the weight of increases in future extraction costs is lower, hence the scarcity rent is lower, as is the resource price, and the extraction rate is higher. Given the higher extraction rate, depth and thus also resource price increase faster in our model, so the growth rate of resource extraction and aggregate production is lower. What distinguishes the present model from the simple Hotelling model is the way that increasing short-run extraction leads to a faster increase in depth, pushing up prices and braking both the increase in extraction and the increase in prices.

\section{Solving the model in more complex cases}

Study of the actual nature of resource stocks and associated extraction costs (next section) shows that the case with $\psi>0$ is empirically relevant; that is, marginal stocks may tend to increase with increasing depth (or decreasing grade) rather than decreasing. However, since total stocks must be finite there must come a point at which marginal stocks decline with depth, or there may be an upper limit on the depth at which resource stocks exist. Furthermore, there may be a limit on the depth at which extraction is practical (possible) irrespective of extraction technology. Finally, because substitutes for the resource may be available, it may be optimal to cease extraction even though stocks remain in the ground. The solution methods for all of these problems are closely related, and we therefore begin with the simplest problem, in which there is some depth $r_{x}$ beyond which extraction is impossible. Having solved this problem we consider the more general problem in which the stock is divided into layers each of which has different parameters. The two types of case are illustrated in Figure 5.

\footnotetext{
${ }^{11}$ Note also that if we choose parameter values such that the inequality in Proposition 2 is reversed then the loci of $\dot{a}$ and $l_{x}^{*}$ approach each other asymptotically as $a \rightarrow \infty$ and $l_{x}^{*} \rightarrow 0$, and on the optimal path we also have $a \rightarrow \infty$ and $l_{x}^{*} \rightarrow 0$, and again $\dot{\lambda} / \lambda=\dot{p} / p=\rho$.
} 


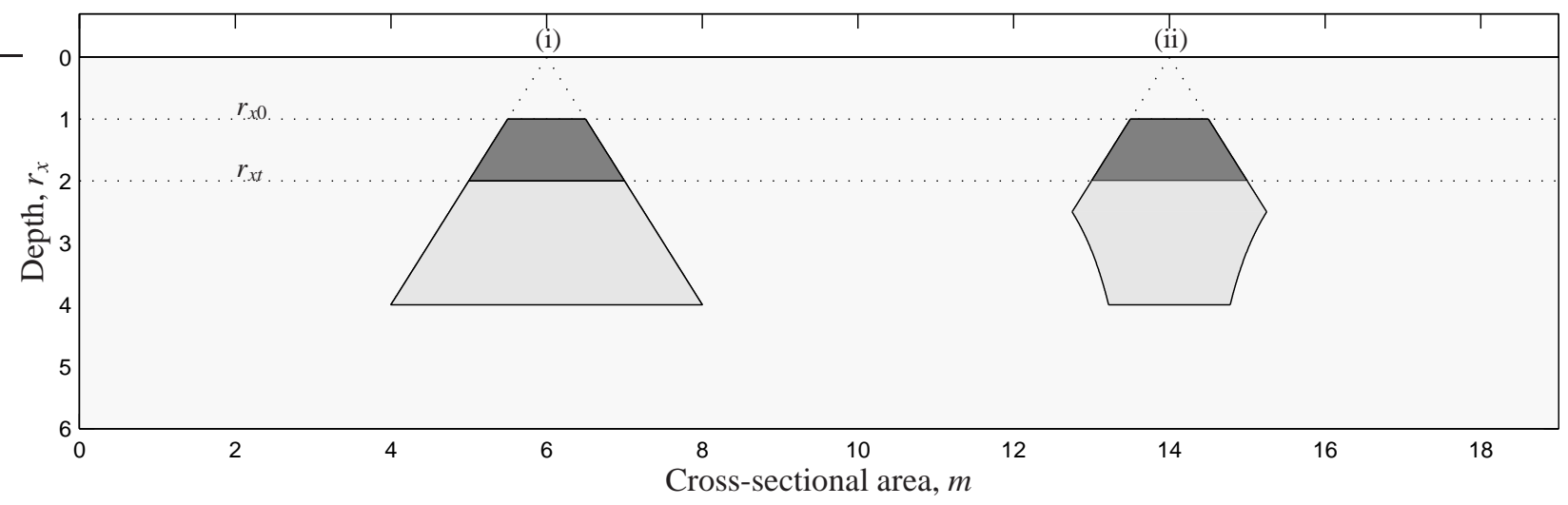

Figure 5: The relationship between depth $r_{x}$ and cross-sectional area $m$ for two alternative resource stocks. In (i) we have $\phi=2$ and no stocks (or inaccessible stocks) for $r_{x}>4$. In (ii) we have $\phi=2$ for $r_{x}<2.5$, and $\phi=0$ for $r_{x}>2.5$, and (again) no stocks for $r_{x}>4$. The dark shading represents cumulative extraction at time $t, n_{t}$, and the lighter shading represents remaining resources at time $t$.

\subsection{When $a_{n}$ is bounded}

Assume there is some depth $r_{x}$ beyond which extraction is impossible, either because of physical constraints on the extraction technology, or because no resource stocks exist beyond this depth. This leads to an upper bound on $a_{n}$, economic depth. When we put an upper bound on $a_{n}$ there is no effect on the expression for the Hamiltonian, and hence no effect on the necessary conditions for optimization stated in Proposition 1, and no effect on the phase diagram. The effect is simply to change the transversality condition, and the result of this change is that the mature b.g.p. is no longer the long-run optimum; this is to be expected, since the bound on $a_{n}$ would be violated if the economy stayed on that b.g.p. indefinitely.

If we continue to assume that there is no substitute for the natural resource input then transversality implies that the resource must be used up asymptotically as time approaches infinity; a greater rate of resource use would violate the stock restriction, whereas a lower rate would leave resources in the ground unnecessarily. In the limit-as the extraction rate approaches zero- $-a_{n}$ is again constant, as it is in the limit of the initial extraction phase moving backwards in time. When $a_{n}$ is constant while $a_{x}$ continues to grow and $x \rightarrow 0$, then $l_{x} \rightarrow 0$ (equation 2 ). Now verify from (4) and (5) that when $l_{x} \rightarrow 0, w l_{x} /(p x) \rightarrow 0$, hence (from 9) in the limit $p=\lambda$, implying (equation 12) that

$$
\dot{\lambda} / \lambda=\dot{p} / p=\rho .
$$

We thus have a third growth path, in addition to the initial and mature growth paths characterized above, which is (in the limit) a simple Hotelling extraction path in which the price is pure rent (hence rising at the discount rate) and extraction costs are zero. Extraction costs approach zero because $a_{n}$ approaches a limit whereas $a_{x} / a_{y}$ grows without bound.

So given the bound on $a_{n}$ the economy approaches the initial path going backwards in time, and the Hotelling path going forwards. What about the mature b.g.p.? The economy will not approach the mature growth path asymptotically; however, the characterization of the mature b.g.p. is still useful. To see this, start by denoting the optimal path given the bound on $a_{n}$ as the bounded path, and denote the optimal path when there is no upper bound on $a_{n}$ as the unbounded path. If the unbounded path is followed even though $a_{n}$ is bounded then at some time $T$ depth $a_{n}$ will reach the bound. Now assume some time $t$ where $t<T$, and consider the bounded path. If $T \gg t$ then (given positive discounting) the difference between the unbounded and the bounded paths will be small, and if we let $T-t \rightarrow \infty$ then the difference must approach zero. So, loosely, as long as resource stocks are sufficiently large the extraction path must first approach the mature path before (as depth starts to approach the bound) moving away from it and approaching instead the Hotelling path.

\subsection{When the stock is divided into layers}

When the stock is divided into layers, as in Figure 5(ii), the optimization problem is divided into sections. Because of discounting, it remains true that resource deposits will be extracted in order of increasing depth, and the first-order 
conditions derived above apply at all times: we are again left with the problem of transversality. The solution is straightforward, as shown by the following proposition.

Proposition 3. At the boundary between layers of the stock, as long as $m$ is a continuous function of $a_{n}$, the initial values of $\left(a, l_{x}^{*}\right)$ for the deeper layer are uniquely determined by the values of $\left(a, l_{x}^{*}\right)$ at the time of exhaustion of the upper layer. The optimal path is then the one for which the initial choice of the control variable $l_{x}^{*}$ for the uppermost layer of the stock leads to satisfaction of the transversality condition for the deepest layer.

Proof. We need to prove that the initial choice of control variable $l_{x}^{*}$ determines the entire extraction path. We know that this choice determines the path up to exhaustion of the first layer, but does it determine the initial values of $\left(a, l_{x}^{*}\right)$ for the second layer? If it does, induction proves that the entire path is determined.

Regarding $a$, since the time of the transition is known (fixing $a_{x t}$ ), and the characteristics of the stock are known, the value of $a$ when extraction from the second layer begins is determined. Regarding $l_{x}^{*}$, note that the transversality condition between layers implies that price must be a continuous function of time; there cannot be a price discontinuity across a boundary between layers, since this would imply non-optimal behaviour. ${ }^{12}$ This implies that the extraction rate $x$ must also be a continuous function of time, since the extraction rate is a continuous function of time if and only if the price is a continuous function of time. Furthermore, we know by assumption that the cross-section $m$ is a continuous function of $a_{n}$, including across boundaries between layers. This implies that extraction employment $l_{x}$ must also be a continuous function of time, i.e. extraction labour does not change discontinuously at the boundary between layers; if $l_{x}$ did change discontinuously, then the extraction rate would change discontinuously, which we ruled out above. Hence initial extraction labour for the second layer is determined by final extraction labour for the first layer.

\subsection{When there is a backstop input}

Finally, assume that there is a backstop input available at an exogenous price, and that there is an upper bound on $a_{n}$. Furthermore, assume (for simplicity) that the backstop price is high in the sense that the backstop price is not reached until the resource is exhausted, at which point the scarcity rent is strictly positive. Now the transversality condition states that the resource price at the point of exhaustion is equal to the price of the backstop. ${ }^{13}$ In terms of the phase diagram, the initial level of $l_{x}^{*}$ (given the initial state $a$ ) is chosen such that exhaustion occurs when $p=\bar{p}$; and when the starting point is determined, the evolution of the system is determined by the dynamic equations. Again, if $\bar{p}$ is sufficiently high then the resource price will be almost pure rent in the run-up to exhaustion, hence the price will rise at close to the discount rate and resource extraction will decline.

In Figure 6 we show the paths of price and economic depth using the same parameters as previously but adding a fixed backstop price $\bar{p}$ and a limit on economic depth $\bar{a}_{n}$. Note the three distinct growth paths - initial, mature, and Hotelling — in accordance with the analytical model: the slopes of $\ln p$ in the three cases are (i) $(1-\alpha)\left(\theta_{a y}-\theta_{a x}\right)$; (ii) $(1-\alpha)\left(\theta_{a y}-\theta_{a x}\right) \psi /(1+\psi)$; and (iii) $\rho$.

\section{Parameterization}

We now turn to the parameterization of the model. The parameterizations are illustrative rather than strictly predictive, the main reason being the great uncertainty concerning many of the assumptions. Nevertheless, the model succeeds in explaining observations from the last 100 years, and makes apparently reasonable predictions for the next several hundred in the cases of oil and copper. We choose these resources based on availability of data and importance of the resources to the global economy.

\footnotetext{
${ }^{12}$ If the price jumps up across the boundary, the upper layer must have been sold too cheaply, whereas if price steps down then the upper layer's price must have been unsustainably high given perfect competition.

${ }^{13}$ The resource price can never be higher than the price of the backstop, whereas if the resource is exhausted with the price lower than the backstop price then resource owners are not extracting the full value of the rent at that point, hence the price path must have been too low.
} 


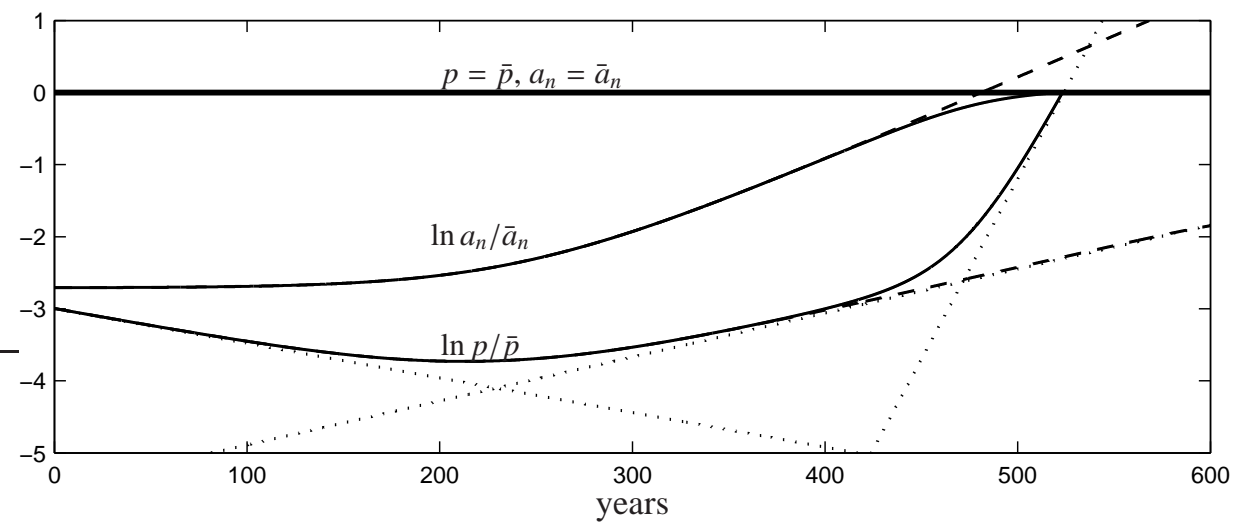

Figure 6: The paths over time of economic depth $a_{n}$ and resource price $p$ (plotted logarithmically and normalized by their final values) when there is a maximum economic depth $\bar{a}_{n}$ and a constant backstop price $\bar{p}$. The dashed lines show the paths of $a_{n}$ and $p$ when depth is unlimited, and the dotted lines have slopes corresponding to the limiting cases of the three phases described above, initial, mature, and exhaustion. Parameters as for Figure 3. Initial state: $a_{x 0}=0.001, a_{y 0}=1, a_{n 0}=1$. Exhaustion: $\bar{a}_{n}=15, \bar{p}=1.1627 \times 10^{4}$.

\subsection{General observations}

We start with some observations which apply whatever the resource for which we are parameterizing the model, in particular the long-run growth rates of TFP, extraction productivity, and the long-run discount rate. For long-run TFP growth we use the estimate of Shackleton (2013) of 1.7 percent per year (1870-2010). ${ }^{14}$ Regarding the productivity $a_{x}$ of extraction labour, it could be argued that this should vary from resource to resource, and should be chosen from case to case in order to fit the data available. However, a more conservative approach is to assume that $a_{x}$ should in the long run track manufacturing productivity generally, regardless of the extraction industry. Thus we take this figure from the literature, hence reducing the degrees of freedom in the parameterization; this reduces the probability that we are able to achieve a spurious match of the model to observations. We take the value of 2.3 percent per year from Fagerberg (2000). We set the discount rate to 5 percent per year. Since manufacturing productivity grows 0.6 percent faster than TFP we expect extraction costs to decline by 0.6 percent per year, ceteris paribus. Finally, $\alpha$ is simply the factor share of the resource.

If we want to match global extraction data we must also consider population growth. We approximate global population growth by assuming a constant growth rate of 1.3 percent per year from 1880-2037, after which population is assumed to be constant; the expected gradual slow-down towards zero growth over the next several decades is thus approximately by a kink in the curve in $2037 .{ }^{15}$ Recall from equation (15) that the effect of population growth $\theta_{L}$ in the model can be captured by increasing the growth rates of $a_{x}$ and $a_{y}$ to $\theta_{a x}+\theta_{L}$ and $\theta_{a y}+\theta_{L}$; since labour is the only input, more labour is equivalent to more productive labour. Note that the combination of the assumptions about TFP and population growth gives a constant growth rate of global product of 3.0 percent per year since 1880, which fits well to the Maddison data used in Figure 1, where the average growth rate since 1900 is 3.0 percent.

\subsection{Extraction and economic depth $a_{n}$}

It remains to find the parameters determining the relationship between cumulative extraction and unit cost for given productivity levels, and the initial values of the variables. How this problem is tackled varies from case to case, hence we discuss our two cases in turn. First however we briefly discuss the general applicability of the model.

The extraction model assumes a link between depth underground $r_{x}$, economic depth $a_{n}=r_{x}^{x}$, and the rate of extraction $x$ for given effective inputs $a_{x} l_{x}: x=a_{x} l_{x} / a_{n}$. However, when applying the model it is not necessary to take the depth of the resource literally. Rather, depth $r_{x}$ should be taken as some observable measure of resource quality, which may typically be a combination of physical depth and grade, as in the case of copper below. The key

\footnotetext{
${ }^{14}$ We define TFP growth as equal to $(1-\alpha) \theta_{\text {ay }}$.

${ }^{15}$ This approximation is broadly in line with UN observations and predictions. See for instance United Nations (1999).
} 
is that cumulative extraction should lead to lower quality resources being exploited over time, and that this change in resource quality should be measurable.

One problem with this approach is the fact that for almost all resources, stocks are divided up into separate deposits, and within-deposit quality varies. Given fixed capital investments at a given site, relatively low-quality resources are likely to be extracted as exhaustion of a given deposit approaches, even though higher-quality resources exist at other sites. A special case of this arises in the case of petroleum, where pressure declines with cumulative extraction from a given deposit, effectively lowering the quality (i.e. raising $r_{x}$ ) of that deposit. However, in the long run we still expect to see a progression from high-quality deposits (low $r_{x}$ ) to low-quality deposits (high $r_{x}$ ). A second problem is that market power leads to extraction of different grades simultaneously, as discussed in the case of petroleum below. See Black and LaFrance (1998) and Cairns and Davis (2001) for analyses of petroleum extraction incorporating geological aspects.

\subsection{Copper}

There are two key dimensions along which the quality of copper deposits varies. The first of these is grade (the fraction of copper in the rock, by mass), and the second is depth. We use Kesler and Wilkinson (2008) as our primary source of data concerning copper resources. They build a model of tectonic migration of copper deposits and calibrate it based on data about known deposits. In practice 'known deposits' is almost synonymous with 'deposits part of [whose] vertical thickness is at the surface' (Kesler and Wilkinson, 2008, p256), and the key to estimating long-run resources is the estimation of sub-surface deposits. Based on the model they estimate a recoverable resource of $8.9 \times 10^{10}$ tons of copper, down to a depth of $3.3 \mathrm{~km}$ (below this depth they assume that recovery is not possible). Furthermore, marginal quantity increases with depth, up to $2.8 \mathrm{~km}$. Regarding grade, Gerst (2008) argues that the grade-tonnage density function is log-Gaussian (his equation 7). Finally, Harmsen et al. (2013) estimate that 85 percent of produced copper historically has come from the top 500 metres. We use these four pieces of informationthe total quantity up to $3.3 \mathrm{~km}$, the relationship between depth and quantity, the grade-tonnage density function, and the depth of historical extraction - to calibrate our model relating $r_{x}$ to $n$.

The first problem is to calibrate equation (18), the relationship between depth $r_{x}$ and cross-sectional area $m$. We do this in two stages. First we estimate a curve showing the relationship between $r_{x}$ and $m$ (without restrictions on the functional form), then we calibrate equation (18) to fit the curve. To estimate the curve we divide the total stock into 7 layers (indexed 1 to 7 with increasing depth) each 471 metres thick, and then use Figure 2 from Kesler and Wilkinson (2008) to divide the total stock of $8.9 \times 10^{10}$ tons into the fractions shown in Table 1. Regarding grade, we assume that the global grade-tonnage density function in each layer is in accordance with the estimate of Gerst (2008) for global porphyry (his Table 3). Regarding the effect of going down one layer, assume a deposit of grade $g$, layer $l$. Then we define the depth (or remoteness) of that deposit as $r_{x}=100 /\left(g \cdot 2^{l-1}\right)$. Thus a step down from one layer to the next is equivalent to a halving of the grade, and extraction costs for a deposit of grade 0.5 percent in the top layer are the same as extraction costs for a deposit of grade 1 percent from the second layer. This is consistent with the observation of Harmsen et al. (2013) that extraction is starting from the second layer at the same time as available stocks in the top layer have dropped from 2 percent to 1 percent grade. For the matlab program for deriving the relationship between depth and cumulative extraction (and thus also the equations and parameter values) see Appendix A.4; the relationship itself is shown by the continuous lines in Figure 7, where the left-hand panel focuses on the most accessible stock, whereas the right-hand panel shows the entire stock.

\begin{tabular}{rccccccc}
\hline Depth $(\mathrm{m})$ & $0-471$ & $471-943$ & $943-1414$ & $1414-1886$ & $1886-2357$ & $2357-2829$ & $2829-3300$ \\
Fraction of total & 0.10 & 0.12 & 0.14 & 0.15 & 0.16 & 0.17 & 0.16 \\
\hline
\end{tabular}

Table 1: Fraction of total available stock to be found at different depths.

The next task is to calibrate equation (18) in order to fit the curve in Figure 7 . In order to match the form of the grade-cumulative stock relationship we divide the stock into two substocks for which different parameters apply: the high-grade substock, consisting of $2.35 \times 10^{9}$ tons of copper, has the following parameters: $r_{x 0}^{*}=50, \phi=4.66$, and $m_{0}=0.0017$; so in 1900, surface copper at 2 percent grade is available, in according with Gerst (2008). The low-grade substock, consisting of $86.7 \times 10^{9}$ tons of copper, has the following parameters: $r_{x 0}=142, \phi=0.25$, and 

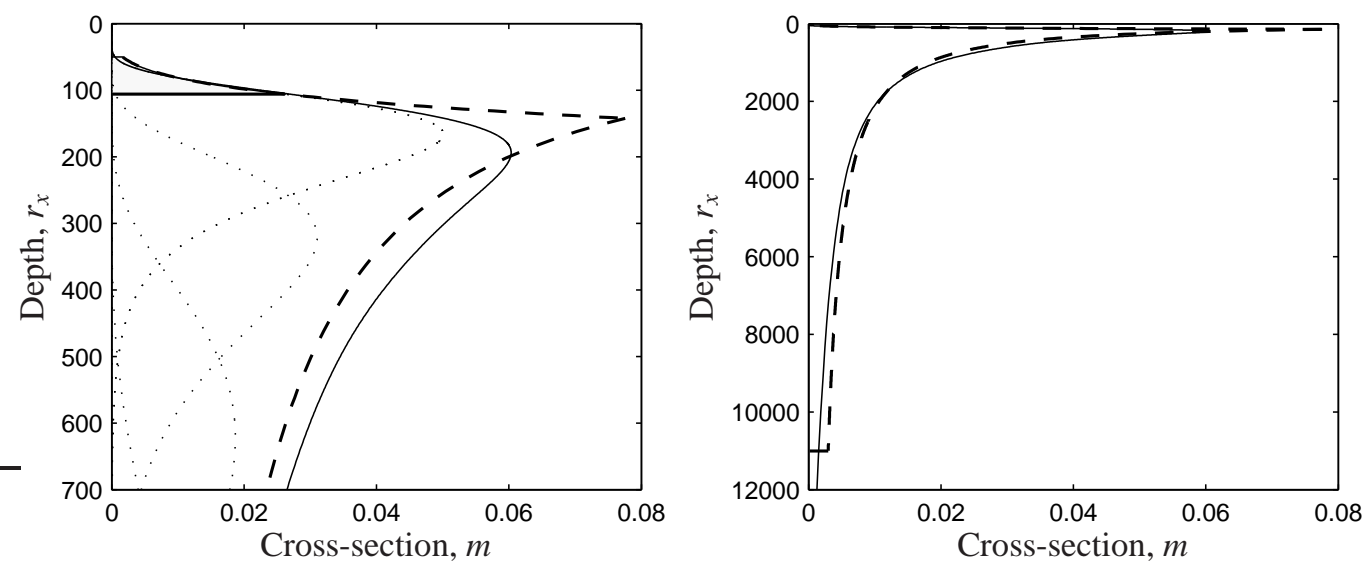

Figure 7: The relationship between the combined measure of depth and grade, $r_{x}$, and cross-sectional area $m$ for copper, based on our interpretation of the literature (continuous line), and the parameterization of our economic model (dashed line). Notice the difference in scale on the two panels. The shaded area shows extraction from 1900-2011, $5.75 \times 10^{8}$ tons, and the dotted lines show the relationship between depth and cross-section layer-by-layer.

$m_{0}=0.0776$. As we can see from Figure 7, the economic model stock fits our stock from the physical model quite well. A much better fit could be achieved by dividing the stock into three parts, each with different parameters.

Having fitted these curves, the final task is to parameterize the relationship between $r_{x}$ and $a_{n}$, i.e. to choose the parameter $\chi$ (recall that $\psi=\phi / \chi$ ). To set the value of $\chi$ we assume that the economy was close to the 'mature' b.g.p. in 1900 , implying that the growth rate of price should be $\theta_{a y}-(1-\alpha) \psi /(1+\psi) \theta_{a x}$. We approximate the observed rate of price decline over the period as 0.4 percent per year, which gives $\chi=0.419$, implying that unit costs are relatively insensitive to increasing depth or decreasing grade. (When grade is divided by 2 at constant depth, unit cost rises by a factor 1.34 at constant technology.) Given the observation of real price and extraction rate in 1900, this effectively completes the parameterization; the starting values are fixed by these observations.

Given the change in the growth rate of population in 2037, the simulation must be done in three stages, the first of which is from 1900 to 2037, the second of which is from 2037 to the (endogenous) time at which the switch of stock parameters must be made - which turns out to be 2054 - and the third of which continues beyond this point until the depth of 3.3 kilometres is reached and the stock is exhausted. The time of exhaustion is also endogenous, and depends on the backstop price. (For information about the program, see Appendix A.4.) The results are shown in Figure 8.
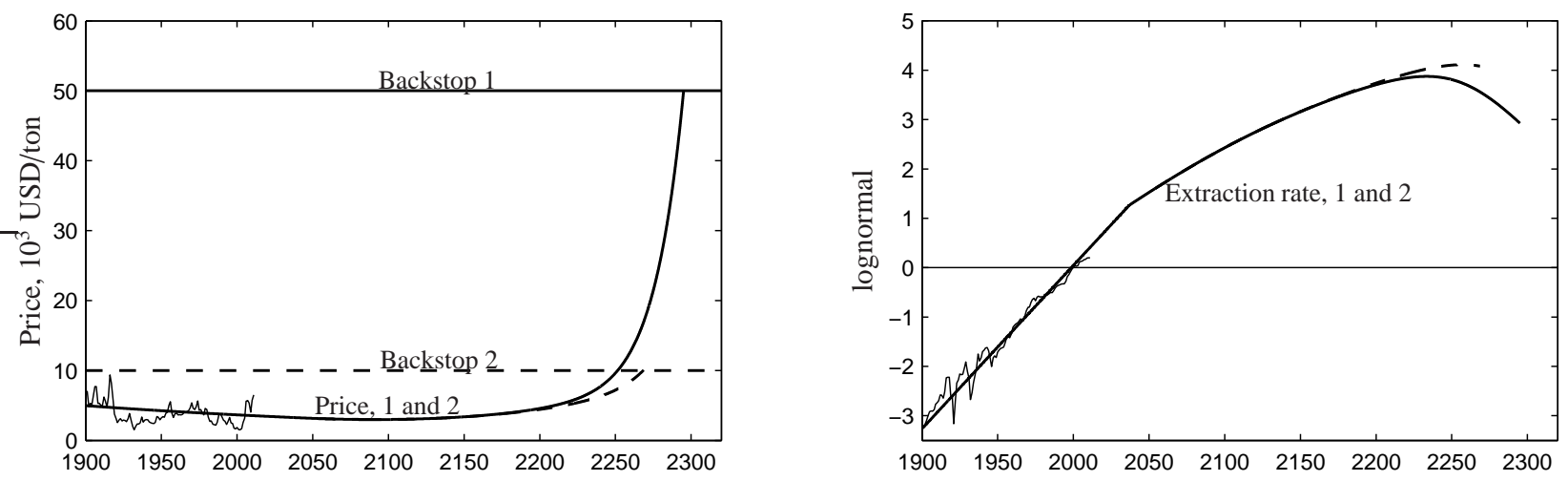

Figure 8: Observed price and extraction rate of copper (thin lines), and the paths of price and extraction rate - up to the time of exhaustion predicted by the model (thicker lines). Two model scenarios are shown, which differ in the assumed backstop price. Note that the extraction rate is plotted on a (natural) logarithmic scale, normalized by the rate in 2000. Prices are in 1998 USD.

To help understand Figure 8 we perform two calculations regarding the behaviour of the economy in the limit when it is on each of the two mature balanced growth paths, firstly for the upper portion of the stock with $\psi=$ $4.66 / 0.419=11.1$, and secondly for the deeper part of the stock for which $\psi=0.25 / 0.419=0.60$. Firstly we 
calculate — using equation (31) — the growth rate of price on the respective b.g.p.s. Since the resource price grows at rate $\theta_{a y}-(1-\alpha) \psi /(1+\psi) \theta_{a x}$, the growth rate of the price is -0.4 percent per year on the first b.g.p., and 0.8 percent per year on the second b.g.p. Secondly we calculate the scarcity rent as a proportion of the total price, using equation (34): on the first the scarcity rent makes up just 5.3 percent of the total price, whereas on the later b.g.p. (which is approached after extraction has moved on to the narrowing section of the stock), the scarcity rent makes up a much larger 40 percent of the total price. This is a reflection of the much higher degradation cost of extraction when the cross-sectional area of the area of the imputed representative resource deposit is declining with depth rather than expanding.

Turning to the figure, the economy starts close to the first b.g.p. which applies for the initial stock, and price declines by around 0.4 percent per year. Once the initial stock is used up in 2054, the rate of increase in depth $a_{n}$ increases, and the economy starts moving towards the second b.g.p. on which price rises by 0.8 percent per year. Finally, from around 2200 the scarcity rent starts to rise as exhaustion approaches, at least in the case with a high backstop price. With a low backstop price the scarcity rent hardly rises, and exhaustion occurs a few years earlier. Note the close agreement between the model and observed trends in prices and extraction rates.

\subsection{Petroleum}

If the copper simulation is an advertisement for the power of the model, the petroleum simulation highlights its weaknesses. There are two key aspects of the petroleum market which the model cannot handle as it stands: firstly, the significance of market power in the petroleum market, and secondly the inextricable links between petroleum and its substitutes, including natural gas, coal, and other energy sources such as nuclear power. Of course, market power and substitutes also exist in the market for copper, but their scale and influence is greater in the oil market. ${ }^{16}$ Concerning market power, consider for instance the fact that petroleum extraction occurs simultaneously from deposits for which marginal extraction costs differ by a factor of 5 or more (compare for instance the Ghawar field in Saudi Arabia to the Athabasca oil sands of Alberta). Concerning substitutes, petroleum demand is linked tightly to markets for coal and other energy sources, and strongly affected by technological change. Consider for instance the substitution from coal to oil driven by the development and refinement of the internal combustion engine. Given these problems-which are evident in Figure 10 - the model calibration is at best illustrative, showing possible future scenarios and highlighting the effect of backstop energy sources.

The data regarding petroleum resources in the ground are uncertain. Furthermore, the data regarding the cost of extraction of these resources are even more uncertain. The most frequently cited paper on the subject is probably Rogner (1997). However, Rogner's curve relating cumulative extraction to extraction cost (see for instance his Figure 6) shows estimated extraction cost at the time of extraction. Its calculation must therefore involve (implicit or explicit) calculations of (i) current extraction costs, (ii) expected decline in extraction costs, and (iii) expected rate of extraction. Since we model the latter two, we need data on the first factor alone, i.e. unit extraction costs for each type of deposit making up the reserves, if full-scale extraction were to be carried out today. This is estimated by the International Energy Agency in their World Energy Outlook 2008 (p.218). The data are very approximate, but can be broadly summarized as follows: considering initial resource stocks, there was a large rather homogeneous stock of easily accessible stocks, approximately 2000 billion barrels at an economic depth of around 18 USD/barrel. Regarding the remaining stocks - about 7000 billion barrels — economic depth $a_{n}$ rises approximately linearly with cumulative extraction, reaching approximately $115 \mathrm{USD} /$ barrel for the deepest stocks. We capture this in the model by assuming an initial stock with low $\psi(\psi=-2.2)$, so that the entire near-homogeneous stock is at a depth of 10-20, switching to the deeper stock with $\psi=1$ from 20-115. The cross-section of the second stock is determined by its size (assumed to be $6.7 \times 10^{9}$ barrels), and the parameters for the first stock are then fixed by the limits on depth (10-20), the size $\left(2.3 \times 10^{9}\right.$ barrels $)$, and the need for $m$ to be continuous over the boundary between the stocks. The result is shown in Figure 9. Note that the curve shows unit extraction costs, in 2008 USD with today's technology, for all petroleum resources including the (hypothetical) current extraction cost of resources already extracted. (Note that we ignore the fact that a significant proportion of cumulative extraction has been from deeper stocks.)

Having fixed the curve in Figure 9, the data on initial price and extraction rate (in 1880) is sufficient to determine the starting value of $a_{x}$, given that we assume that the economy starts with a low value of $a$ and hence $l_{x}$ close to $\alpha$.

\footnotetext{
${ }^{16}$ For recent work on market power in these markets see Lin (2009), Lin Lawell and Zhang (2015) and Lin Lawell (2016).
} 


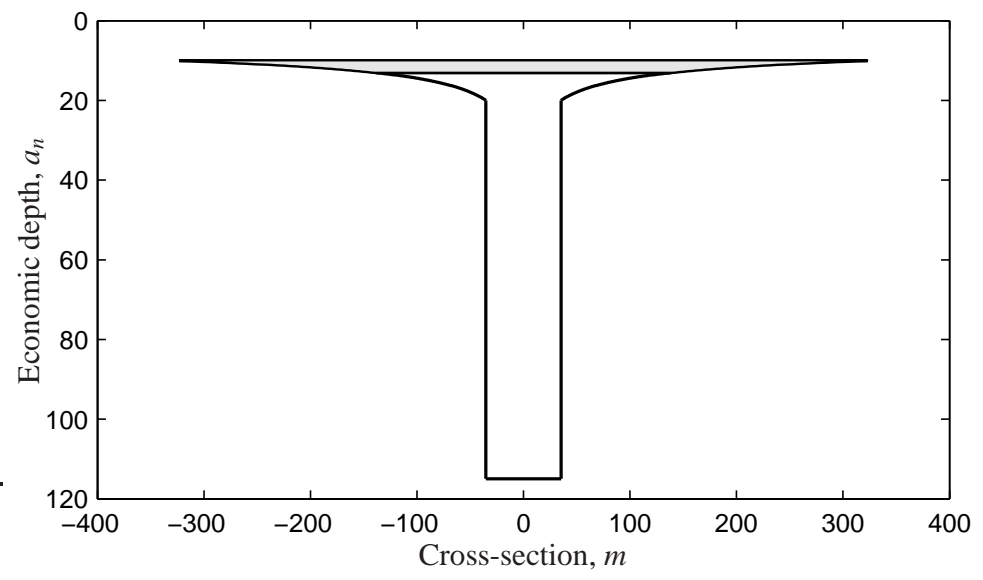

Figure 9: The relationship between economic depth, $a_{n}$, and cross-sectional area $m$ for oil, based on our interpretation of the International Energy Agency World Energy Outlook 2008. Depth is measured in 2008 USD, and cross-sectional area in billion barrels per USD. The shaded area shows extraction from 1900-2008, 1100 billion barrels.

(Recall that $\alpha$ is the share of petroleum in global product, which we set at 3 percent.) Unfortunately, however, this parametrization fails to reflect the fact that the share of petroleum in global product rose rapidly from 1880 to 1970 , contrary to the assumption of constant share in the model. The problem is that although the share of combustibles in global product show no long-run trend over this period (recall Figure 1), petroleum substituted for other combustibles and hence its share rose. ${ }^{17}$ To account for this in the model we raise the productivity growth rates during the period up to 1970, reflecting petroleum's increasing market penetration.

Having parameterized the model, and given the assumption about the total stock of resources, the future development of prices and quantities predicted by the model depends on what we assume about the price of the backstop (i.e. the substitutes for oil that will take over when oil is exhausted or too expensive). Here we make two alternative assumptions to demonstrate the role played by the backstop resource. In the first case we assume that a backstop is available at a fixed price of 150 US dollars (2011); in the second case we assume that a backstop is available today at that price, and that this price will decline at the rate $\theta_{a x}-\theta_{a y}$; that is, the backstop price declines as long as manufacturing productivity growth outstrips TFP growth. The result is that the backstop price is around 65 USD at the time of exhaustion, rather than 150 .

Consider now Figure 10. Recall that there is no market power in the model economy, hence the results are what the model predicts in an economy similar to the actual global economy but without the exercise of market power by oil producers; this implies that where observed price is well above that predicted by the model, a possible explanation is the exercise of market power, an explanation supported by the analysis of Lin (2009). Turning now to the results, up to the exhaustion of the upper stock, depth is almost constant, the scarcity rent is close to zero, and price declines at a rate equal to the difference between the growth rates of extraction productivity and labour productivity in final-good production, i.e. 0.6 percent per year (in accordance with equation 32). However, as the upper stock nears exhaustion depth starts to rise at a significant rate, and the economy heads back towards the b.g.p. for the stock, for which $\psi=1$; the mature extraction phase. On this b.g.p. we have - from equations (33)-(34) — that the growth rate of extraction is halved, the resource price rises by 0.6 percent per year, and the scarcity rent makes up 21 percent of the price. ${ }^{18}$

In the latter half of the 21 st century the price paths of the alternative backstop scenarios diverge significantly: the upper path (high backstop price) is slightly above the b.g.p. price path, while the lower path is below it. Hence when the backstop price is fixed at 150 USD the scarcity rent rises above 21 percent of the total price as exhaustion approaches, whereas given the lower backstop price the rate of price increase slows down as exhaustion approaches, and the rent actually declines as a proportion of the price.

\footnotetext{
${ }^{17}$ The share also rose after 1974 , but this was due to short-run inelasticity of demand combined with steeply rising prices.

${ }^{18}$ Note that after the transition to the deeper stock with $\psi=1$ the economy approaches the mature b.g.p. for that stock from above, i.e. the state variable $a$ is above its level in the steady state. As the economy approaches the new b.g.p. $a$ falls back, which is why prices rise quite steeply throughout the 21 st century.
} 

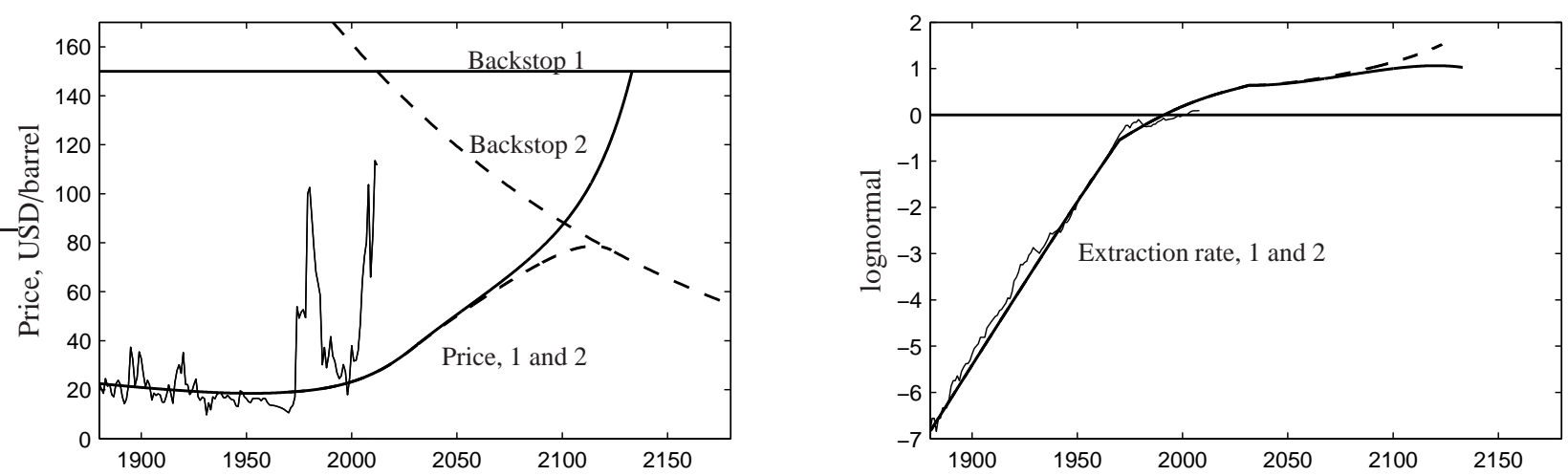

Figure 10: Observed price and extraction rate of petroleum (thin lines), and the paths of price and extraction rate-up to the time of exhaustion predicted by the model (thicker lines). Two model scenarios are shown, which differ in the assumed backstop price. Note that the extraction rate is plotted on a logarithmic scale, normalized by the rate in 2000. Prices are in 2012 USD. Scenarios: continuous lines, backstop price 150 USD (year 2012); dashed lines, current backstop price 150 USD, declining at a rate $\theta_{a x}-\theta_{a y}$ per year. Price data from BP (2012), consumption data from Boden et al. (2012), assuming a linear relationship between $\mathrm{CO}_{2}$ emissions and petroleum consumption.

\subsection{Sensitivity to assumptions}

Clearly the above simulations are sensitive to the assumptions made, the most uncertain of which are those regarding future demand, and future development of extraction productivity. On the one hand, our assumption about future demand is essentially at the upper bound of what is realistic, i.e. that demand per capita continues to grow indefinitely at a similar rate to the rate observed over the last 100 years. At least two factors might be expected to lead to lower future demand: firstly, if global growth slows in the long run, and secondly if there is a transition from 'early' growth based on manufacturing and hence resources, and 'post-industrial' growth based on services and hence labour. ${ }^{19}$ The effect of lower demand would be to reduce extraction rates and hence also reduce the growth rate of prices predicted by the model. On the other hand, our assumption regarding future development of extraction productivity is also an upper bound; again, we assume that it continues to increase indefinitely. This is unlikely, not least because in reality resource extraction requires energy, and there are physical limits to the efficiency with which this energy can be used. Since these limits are already coming close in some cases, this implies that even if labour productivity continues to increase, energy productivity will not do so and hence the proportion of the energy cost in the unit cost will rise, and the rise of overall extraction productivity will slow. This assumption therefore biases the results towards lower prices and higher extraction rates than are likely to be observed.

The effect of assuming both lower future demand and lower productivity growth rates in extraction is therefore that the price path is likely to be relatively unchanged, whereas the extraction path will be lower. Furthermore, the proportion of the price accounted for by the scarcity rent will be lower. Given a finite stock, the lower extraction path will lead to later exhaustion, and hence any price spike as exhaustion approaches is also likely to be delayed.

\section{Conclusions}

We show how the problem of optimal extraction of inhomogeneous resource deposits can be set up in such a way that an analytical solution for the equilibrium extraction path can be obtained. The model provides an explanation for the historical observation of slowly declining resource prices - the productivity of the extraction input grows faster than its price, since extraction productivity grows faster than total factor productivity in the final-good sector-and also predicts a more constant or slowly rising price trend in the medium term, as the above effect is supplemented

\footnotetext{
${ }^{19}$ To get a feel for the sizes of demand changes in the model, we consider each simulation in turn. For copper, the extraction rate peaks at around 50 times the observed rate in year 2000. Compare this to the arbitrary assumption that the entire future global population consumes copper at the same rate as the average U.S. citizen in year 2000; this would lead to a global extraction rate approximately 6.3 times greater than that observed in 2000. If demand levels off in this way then the copper stocks will last for many centuries rather than just two or three. For petroleum, the extraction rate in the model peaks at around 3 times the year 2000 rate, which is less than the rate which would arise if all countries matched the U.S. per-capita rate from year 2000.
} 
by the effect of the increasing depth from which resources must be extracted. Finally, if exhaustion beckons and substitutes are very expensive there may be a third phase in which price rises at a rate approaching the discount rate while the extraction rate declines.

We study just two resources empirically. It would be useful to apply the model to analysis of further resources, both as a test of the model and to get further results. To give some perspective on the relevance of resource limits in general consider the following calculation. The current physical extraction rate of minerals is of the order of $10^{10}$ tonnes per year globally according to the Kelly and Matos (2012) data, which is around $10^{-7}$ percent of the earth's crust (based on a figure of $2 \times 10^{19}$ tons for the Earth's crust). Now assume that the extraction rate continues to grow as it has done over the past 100 years, i.e. by approximately 3 percent per year. Then extraction would be multiplied by a factor 20 each century, and in 700 years we would be mining and using minerals roughly equal to the entire earth's crust every year. Clearly we live in an exceptional - and temporary - period of rapidly expanding resource extraction.

The model is highly simplified. Better data about the nature of resource stocks, and a better understanding of long-run demand for resources (in particular whether increasing resource extraction is primarily driven by relative price effects or by income effects) would allow the model to be refined and increase confidence in the predictions. Another relatively straightforward extension would be to include other inputs — such as capital and energy — into the extraction cost and production functions; this might be particularly important in the extraction sector, which is capitaland energy-intensive, potentially providing a more detailed explanation of why extraction productivity grows faster than productivity in the final-good sector. ${ }^{20}$ An extension with more subtle consequences would be to generalize the utility function to allow the discount rate to vary over time. A general productivity slow-down, or a scarcity-induced crisis, would then affect the discount rate and via this channel also the evolution of the scarcity rent. Finally, to account properly for resource markets in which the exercise of market power is a crucial factor-such as the market for petroleum - a major extension to the model would be required. Nevertheless, the model as it stands supports the view that the high price of petroleum is neither due to extraction costs nor scarcity rent, and hence is presumably due to market power.

\section{Acknowledgements}

Thanks to all the referees who made constructive comments on previous versions. Thanks especially to Sjak Smulders, Geir Asheim, and Luca di Corato for help and encouragement, and to numerous seminar and conference participants. The work was supported by Formas, project number 2007-1323. Formas had no involvement in the research.

\section{Appendix A Additional results, proofs, and material}

\section{A.1 The solution for equation (15)}

Take equations (2), (4), (5), the labour restriction, and (6): $x_{t}=l_{x t} a_{x t} / a_{n t} ; w=(1-\alpha) y /\left(L-l_{x}\right) ; p=\alpha y / x$; $L=l_{x}+l_{y} ; y=a_{y}^{1-\alpha}\left(L-l_{x}\right)^{1-\alpha} x^{\alpha}$. Differentiate each with respect to $t$ to yield

$$
\frac{\dot{w}}{w}=\frac{\dot{y}}{y}-\frac{\dot{l}_{y}}{l_{y}} ; \quad \frac{\dot{p}}{p}=\frac{\dot{y}}{y}-\frac{\dot{x}}{x} ; \quad \frac{\dot{y}}{y}=(1-\alpha)\left(\theta_{a y}+\frac{\dot{l}_{y}}{l_{y}}\right)+\alpha \frac{\dot{x}}{x} ; \quad \frac{\dot{l}_{y}}{l_{y}}=\theta_{L} \frac{L}{l_{y}}-\frac{\dot{l}_{x}}{l_{x}} \frac{l_{x}}{l_{y}} ; \quad \frac{\dot{x}}{x}=\frac{\dot{l}_{x}}{l_{x}}+\theta_{a x}-\frac{\dot{a}_{n}}{a_{n}} .
$$

Substitute each of these equations, in turn, into equation (14), $\dot{p} / p=\rho(1-\gamma)+\left(\dot{w} / w-\theta_{a x}\right) \gamma$, and use the definition of $\gamma\left(\gamma=w l_{x} /(p x)\right)$ to yield (15).

\footnotetext{
${ }^{20}$ If we continue to assume labour-augmenting technological progress and keep the interest rate fixed then it is straightforward to show that introducing capital makes no difference, even if the extraction sector is assumed to be more capital-intensive. However, if we allowed for increases in capital-augmenting knowledge as well as labour-augmenting then we would have a mechanism explaining why TFP increases faster in the extraction sector than in the final-good sector.
} 


\section{A.2 The transversality condition}

Our proof of the sufficiency of the transversality condition (29) is based on Acemoglu (2009), Theorem 7.14, as follows. Recall that we already have the current-value Hamiltonian and necessary first-order conditions in $x$ and $n$. Having defined $F$ we add the transversality condition, and find a solution consistent with the conditions. Then we note that the value of the Hamiltonian along the optimal path - in Acemoglu's notation, $M(t, n, \lambda)$ - is simply equal to zero; this follows from the first-order condition in $x$, because of the linearity of the Hamiltonian in $x$. Since $M \equiv 0$ it follows that $M$ is concave in $n$ for all $t$. Then since the set of allowed values for $n$ is convex, and if there are no discontinuities, the solution must achieve the global maximum.

Now we prove that only the stable path is consistent with the transversality condition. Consider first the corner with $l_{x}=0$ and $a \rightarrow \infty$. As we head into this corner $\lambda$ approaches $p$ (equation 9). So $\dot{\lambda} / \lambda=\dot{p} / p$. But from (14) we have that $\rho=\dot{p} / p$ in this corner, so (putting these results together) we have $\dot{\lambda} / \lambda=\rho$. Hence the transversality condition cannot be satisfied; valuable resources are unnecessarily left in the ground. Now to the corner with $l_{x}=L$. Use (4) and (6) to show that when $l_{x} \rightarrow L, w \rightarrow \infty$, implying (9) that $\lambda \rightarrow-\infty$; loosely speaking, infinitely-priced labour is being devoted to extracting resources which are then not usable because there is no production labour. Finally, it is straightforward to show that the path leading to the steady-state satisfies the transversality condition, using equations (31) and (33). (Note that when $\psi>0$, then $\dot{n} / n \rightarrow \dot{x} / x$ as $t \rightarrow \infty$, whereas when $\psi<0$ then $\dot{n} / n \rightarrow 0$ as $t \rightarrow \infty$.)

\section{A.3 The mature b.g.p.}

To show that (33) holds in the steady state of the transformed system take (24) and use it to show that when the growth rates of $a$ and $l_{x}$ are zero then

$$
\theta_{a x}=(1+\psi) \dot{a}_{n} / a_{n} \text { and } \dot{x} / x=\theta_{a x}-\dot{a}_{n} / a_{n},
$$

hence $\dot{x} / x=\psi /(1+\psi) \theta_{a x}$, i.e. (33). Now take (9) and use it to show that $\lambda / p$ is constant if $\dot{w} / w+\dot{a}_{n} / a_{n}=\dot{p} / p+\theta_{a x}$, and verify that the latter equation holds on the b.g.p. using the two expressions above and (31).

To find the levels of the variables on the b.g.p., use the growth rate of $a_{n}$ on the b.g.p. $-\dot{a}_{n} / a_{n}=\theta_{a x} /(1+\psi)-$ and (23) to show that

$$
\frac{\theta_{a x}}{1+\psi}=\frac{x}{F_{0}\left(a_{n} / a_{n 0}\right)^{\psi}}
$$

Rearrange and substitute for $a_{n}$ using (2) to derive

$$
x=\left(\frac{a_{x} l_{x}}{a_{n 0}}\right)^{\psi /(1+\psi)}\left(\frac{F_{0} \theta_{a x}}{1+\psi}\right)^{1 /(1+\psi)} .
$$

The value of $l_{x}$ follows directly from (27) and (28):

$$
\frac{l_{x}}{\alpha L}=\frac{\frac{\psi}{1+\psi} \theta_{a x}-\theta_{a y}+\frac{\rho}{1-\alpha}}{\theta_{a x}-\theta_{a y}+\frac{\rho}{1-\alpha}} .
$$

Now use (2), (4), (5), and (9) to show that $\lambda / p=1-(1-\alpha) / \alpha \cdot l_{x} /\left(L-l_{x}\right)$. Insert the expression for $l_{x}$ derived above to yield the expression for $\lambda / p$ in (34).

\section{A.4 Supplementary material regarding the solutions to the models and the figures}

All of the programs used to solve the models and generate the figures (including data) are to be found on the following site: https://sites.google.com/site/exresmat/.

Acemoglu, D., 2009. Introduction to modern economic growth. Princeton, New Jersey.

André, F. J., Smulders, S., 2014. Fueling growth when oil peaks: Directed technological change and the limits to efficiency. European Economic Review 69, 18-39.

Arrow, K. J., Chang, S., Mar. 1982. Optimal pricing, use, and exploration of uncertain natural resource stocks. Journal of Environmental Economics and Management 9 (1), 1-10.

Black, G., LaFrance, J., 1998. Is Hotelling's rule relevant to domestic oil production? Journal of Environmental Economics and Management $36(2), 149-169$ 
Boden, T. A., Marland, G., Andres, R. J., 2012. Global, regional, and national fossil-fuel $\mathrm{CO}_{2}$ emissions. Tech. rep., CDIAC, Oak Ridge National Laboratory, U.S. Department of Energy, Oak Ridge, Tenn., U.S.A.

BP, 2012. Statistical review of world energy. Annually published data, British petroleum.

Cairns, R. D., Davis, G. A., 2001. Adelman's rule and the petroleum firm. The Energy Journal 22 (3), 31-54.

Dasgupta, P., Heal, G., 1974. The optimal depletion of exhaustible resources. The Review of Economic Studies 41, 3-28.

Dasgupta, P., Heal, G., 1979. Economic Theory and Exhaustible Resources. Cambridge University Press, Cambridge.

Di Maria, C., Smulders, S., van der Werf, E., Feb. 2012. Absolute abundance and relative scarcity: Environmental policy with implementation lags. Ecological Economics 74, 104-119.

Ellis, G. M., Halvorsen, R., Aug. 2002. Estimation of market power in a nonrenewable resource industry. Journal of Political Economy 110 (4), 883-899.

Fagerberg, J., Dec. 2000. Technological progress, structural change and productivity growth: a comparative study. Structural Change and Economic Dynamics 11 (4), 393-411.

Farzin, Y. H., Jul. 1992. The time path of scarcity rent in the theory of exhaustible resources. Economic Journal 102 (413), 813-830.

Fouquet, R., 2011. Divergences in long-run trends in the prices of energy and energy services. Review of Environmental Economics and Policy 5 (2), 196-218.

Gerst, M. D., May 2008. Revisiting the cumulative grade-tonnage relationship for major copper ore types. Economic Geology 103 (3), $615-628$.

Goeller, H. E., Weinberg, A. M., 1978. The age of substitutability. American Economic Review 68 (6), 1-11, reprinted from Science 191 pp. $683-689,1976$

Groth, C., 2007. A new-growth perspective on non-renewable resources. In: Bretschger, L., Smulders, S. (Eds.), Sustainable Resource Use and Economic Dynamics. Springer, pp. 127-163.

Hanson, D. A., 1980. Increasing extraction costs and resource prices-some further results. Bell Journal of Economics 11 (1), $335-342$.

Harmsen, J., Roes, A., Patel, M., Feb. 2013. The impact of copper scarcity on the efficiency of 2050 global renewable energy scenarios. Energy 50 , $62-73$.

Hart, R., Spiro, D., 2011. The elephant in Hotelling's room. Energy Policy 39 (12), 7834-7838.

Hartwick, J. M., 1977. Intergenerational equity and the investing of rents from exhaustible resources. American Economic Review 67, $972-974$.

Heal, G., 1976. The relationship between price and extraction cost for a resource with a backstop technology. The Bell Journal of Economics 7 (2), 371-378.

Hotelling, H., 1931. The economics of exhaustible resources. Journal of Political Economy 39, 137-175.

Kelly, T. D., Matos, G. R., 2012. Historical statistics for mineral and material commodities in the United States. Tech. rep., U.S. Geological Survey, Data Series 140 URL http: //pubs.usgs.gov/ds/2005/140/

Kesler, S. E., Wilkinson, B. H., Mar. 2008. Earth's copper resources estimated from tectonic diffusion of porphyry copper deposits. Geology 36, $255-258$.

Krautkraemer, J., 1998. Nonrenewable resource scarcity. Journal of Economic Literature 36, 2065-2107.

Krulce, D. L., 1993. Increasing scarcity rent - a sufficient condition. Economics Letters 43 (2), 235-238.

Levhari, D., Liviatan, N., 1977. Notes on Hotelling's economics of exhaustible resources. Canadian Journal of Economics - revue Canadienne D Economique 10 (2), 177-192.

Lin, C.-Y. C., 2009. Insights from a simple Hotelling model of the world oil market. Natural Resources Research 18 (1), 19-28.

Lin, C.-Y. C., 2011. Estimating supply and demand in the world oil market. Journal of Energy and Development 34 (1), 1-32.

Lin, C.-Y. C., Meng, H., Ngai, T. Y., Oscherov, V., Zhu, Y. H., 2009. Hotelling revisited: Oil prices and endogenous technological progress. Natural Resources Research 18 (1), 29-38.

Lin, C.-Y. C., Wagner, G., Jul. 2007. Steady-state growth in a Hotelling model of resource extraction. Journal of Environmental Economics and Management 54 (1), 68-83.

Lin Lawell, C.-Y. C., 2016. Market power in the world oil market: Evidence for an OPEC cartel and an oligopolistic non-OPEC fringe. Working paper, University of California at Davis.

Lin Lawell, C.-Y. C., Zhang, W., 2015. Market power in nonrenewable resource markets: An empirical dynamic model. Working paper, University of California at Davis.

Livernois, J., 2009. On the empirical significance of the Hotelling rule. Review of Environmental Economics and Policy 3, $22-41$.

Livernois, J., Martin, P., 2001. Price, scarcity rent, and a modified $r$ per cent rule for non-renewable resources. Canadian Journal of Economics 34 (3), 827-845.

Maddison, A., 2003. Growth accounts, technological change, and the role of energy in Western growth. In: Economia e Energia, secc.XIII-XVIII. Istituto Internazionale di Storia Economica "F. Datini" Prato, Florence.

Maddison, A., 2010. Historical statistics of the world economy: 1-2008 ad. Tech. rep., Groningen growth and development centre.

Mercure, J.-F., Salas, P., Oct. 2012. An assessement of global energy resource economic potentials. Energy 46 (1), $322-336$.

Ploeg, F. v., Withagen, C., Nov. 2012. Is there really a green paradox? Journal of Environmental Economics and Management 64 (3), $342-363$.

Rogner, H. H., 1997. An assessment of world hydrocarbon resources. Annual Review of Energy and the Environment 22, $217-262$.

Shackleton, R., 2013. Total factor productivity growth in historical perspective. Working paper series, CBO $2013-01$.

Sinclair, P. J. N., Oct. 1994. On the optimum trend of fossil fuel taxation. Oxford Economic Papers-New Series 46, 869-877.

Sinn, H.-W., Aug. 2008. Public policies against global warming: a supply side approach. International Tax and Public Finance 15 (4), $360-394$.

Slade, M. E., 1982. Trends in natural-resource commodity prices: An analysis of the time domain. Journal of Environmental Economics and Management 9, 122-137.

Solow, R. M., 1974. Intergenerational equity and exhaustible resources. Review of Economic Studies, Symposium on the economics of exhaustible resources 41, 29-45.

Solow, R. M., Wan, F. Y., 1976. Extraction costs in the theory of exhaustible resources. Bell Journal of Economics 7 (2), 359-370.

Spiro, D., 2014. Resource prices and planning horizons. Memorandum 14/2014, Department of Economics, University of Oslo. 
Stiglitz, J. E., 1974. Growth with exhaustible natural resources: Efficient and optimal growth paths. Review of Economic Studies, Symposium on the Economics of Exhaustible Resources 41, 123-37.

Stiglitz, J. E., 1976. Monopoly and the rate of extraction of exhaustible resources. American Economic Review 66 (4), $655-661$.

Ulph, A., Ulph, D., 1994. The optimal time path of a carbon tax. Oxford Economic Papers 46, 857-868.

United Nations, 1999. The World at Six Billion. Population Division, Department of Economic and Social Affairs, United Nations Secretariat. 